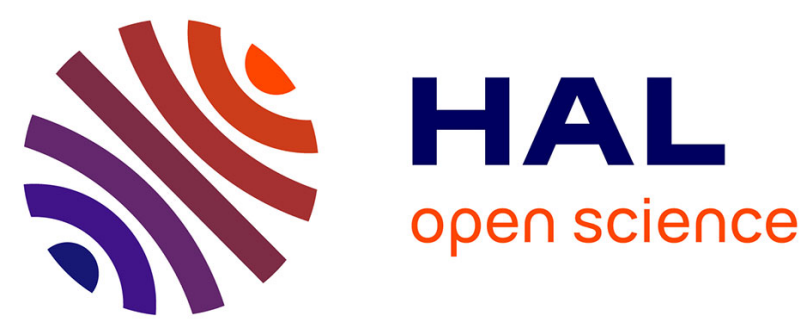

\title{
Mediator, known as a coactivator, can act in transcription initiation in an activator-independent manner in vivo
}

Hélène Jean-Jacques, Siew Lay Poh, Laurent Kuras

\section{- To cite this version:}

Hélène Jean-Jacques, Siew Lay Poh, Laurent Kuras. Mediator, known as a coactivator, can act in transcription initiation in an activator-independent manner in vivo. Biochimica et Biophysica Acta

- Gene Regulatory Mechanisms , 2018, 1861, pp.687 - 696. 10.1016/j.bbagrm.2018.07.001 . hal03448792

\section{HAL Id: hal-03448792 \\ https://hal.science/hal-03448792}

Submitted on 5 Jan 2022

HAL is a multi-disciplinary open access archive for the deposit and dissemination of scientific research documents, whether they are published or not. The documents may come from teaching and research institutions in France or abroad, or from public or private research centers.
L'archive ouverte pluridisciplinaire HAL, est destinée au dépôt et à la diffusion de documents scientifiques de niveau recherche, publiés ou non, émanant des établissements d'enseignement et de recherche français ou étrangers, des laboratoires publics ou privés. 
Mediator, known as a coactivator, can act in transcription initiation in an activator-independent manner in vivo

Hélène Jean-Jacques, Siew Lay Poh and Laurent Kuras*

Institute for Integrative Biology of the Cell (I2BC), CEA, CNRS, Univ. Paris-Sud, Université ParisSaclay, Gif-sur-Yvette, France

*Corresponding author: Institute for Integrative Biology of the Cell (I2BC), Avenue de la Terrasse, 91198 Gif-sur-Yvette cedex, France.

E-mail address: laurent.kuras@i2bc.paris-saclay.fr; Tel. +33169823159 


\section{ABSTRACT}

Mediator is an evolutionarily conserved complex best known for its role as a coactivator responsible for transducing regulatory signals from DNA-bound activators to the basal RNA polymerase II (Pol II) machinery that initiates transcription from promoters of protein-encoding genes. By exploiting our in vivo activator-independent transcription assay in Saccharomyces cerevisiae, in combination with new temperature sensitive (ts) mutants of Med14 N-terminal half exhibiting widespread transcriptional defects, and existing ts mutants of Kin28 and Med17, we show that, in the absence of activator: (i) Mediator can associate with a promoter as a form devoid of the Cyclin-dependent kinase 8 (CDK8) module, and this association remains regulated by Kin28; (ii) Mediator can stimulate the assembly of the entire Pol II initiation machinery. Although the literature emphasizes the role of the interaction between activators and Mediator, together our results support the view that Mediator is able to act through a dual mechanism in vivo, activator-dependent but also activator-independent, therefore not always as a coactivator.

Keywords: transcriptional coactivator; general transcription factor; MED14; MED17; CDK8 


\section{Introduction}

Mediator is a large evolutionarily conserved eukaryotic complex (25-30 proteins), that plays crucial roles in transcription of protein-coding genes [1-3]. It was initially discovered for its ability to act, in acellular transcription assays reconstituted with purified factors, as a coactivator bridging gene-specific DNA-bound activators to the basal transcription initiation machinery consisting of RNA polymerase II (Pol II) and General Transcription Factors (GTFs; including TBP and TFIIB, IIE, IIF, IIH) [4]. The notion that Mediator has a coactivator function is supported by genetic, biochemical and structural studies that demonstrated direct physical interactions between Mediator and various yeast and mammalian activators as well as between Mediator and Pol II or some GTFs [5-7].

The 25-subunit Mediator complex of S. cerevisiae is structured in three core modules (Tail, Middle and Head), along with a fourth dissociable module containing the cyclin-dependent kinase CDK8 (Srb10), which associates dynamically with the core modules [8-10]. Subunits that were found to engage in direct interactions with activators in S. cerevisiae are localized in the Tail module [5], and those in contacts with Pol II and GTFs are localized at the level of the Middle and Head modules [1115]. X-ray crystallography and cryo-electron microscopy (EM) studies have led to elaborate architectural models of the S. cerevisiae Mediator complex, revealing unique architectural roles for the two large essential subunits Med17 (Srb4) and Med14 (Rgr1) [16-20]. Med17 (687 amino acid residues) extends over most of the Head module, making extensive contacts with other subunits within the module, and is involved in the connection with the Middle module as well. Med14 (1082 amino acid residues) extends from the junction between the Tail and Middle modules to the tip of the Middle module and makes contact with subunits of the Head domain, including Med17. Supporting the structural data, the S. cerevisiae med17-138 (srb4-138) temperature-sensitive (ts) allele causes instability of the complex and a general arrest of all Pol II-dependent transcription at restrictive temperature [21-23]. Two studies have recently shown, using distinct depletion methods, that Med14 elimination in $S$. cerevisiae leads to an important genome-wide decrease in Pol II occupancy [24,25]; however, no conditional allele of MED14 has been reported. 
Mediator targeting to gene promoters is assumed to occur through interactions with DNA-bound activators, distinctively from Pol II and the GTFs. This assumption is supported by Chromatin Immunoprecipitation (ChIP) experiments in yeast showing temporal and physical separation at model promoters between recruitments of Mediator and Pol II [26-28]. Moreover maximal ChIP occupancy signals are found at upstream promoter regions for Mediator and at the core promoter for the Pol II initiation machinery, further highlighting the role of activators in the recruitment of Mediator [28-31]. The function of Mediator once recruited by an activator and the detailed underlying molecular mechanisms have been under intense study. There is strong evidence that Mediator acts at least at two different steps during transcription initiation: first by helping to assemble the pre-initiation complex (PIC), presumably through mechanisms involving interplays with chromatin regulators and/or direct contacts with GTFs and Pol II [5,11,32,33]; and second by stimulating phosphorylation of Pol II carboxy-terminal domain (CTD) by the Kin28 kinase subunit of TFIIH, a step which is important to release the interaction between Mediator and Pol II prior to elongation $[4,34,35]$. There is also evidence for an involvement of Mediator at post-initiation stages of transcription, including elongation, particularly in mammals [36].

It has been shown that Mediator can stimulate basal transcription in yeast and mammalian in vitro transcription assays devoid of activator [4,37-39]. However, evidence that Mediator can operate independently of activators within the context of the cell is scarce, and the underlying question of whether the contact with an activator is obligatorily required in vivo, or can be dispensable under some circumstances, is still disputable. Addressing this point represents a challenging task for several reasons, including: first, uninduced (or basal) transcription in vivo corresponds to transcription levels that are too low to consider Mediator occupancy analysis; second, evidence of transcriptionally active genes not bound and controlled by an activator is lacking. To circumvent these problems, we developed in $S$. cerevisiae in our previous studies an activator-independent in vivo transcription assay based on the recruitment of the Pol II machinery to a test promoter through artificial tethering of one GTF [40]. We found that Med5 (Tail) and Med14 (Tail/Middle) were associated with the test promoter when transcription was induced by tethering of TFIIB, showing that Mediator can be targeted to a promoter 
in the absence of activator in vivo. Whether the complete Mediator complex was recruited in our activator-independent transcription assay, or only the core complex without the CDK module, remains to be determined. Two recent studies proposing that Mediator would be first recruited by activators in the complete form, followed by dissociation of the CDK module upon interaction with Pol II and the PIC at the promoter [41,42], raises the question of which form of Mediator is recruited in the absence of activator.

We found in Lacombe et al [40] that inactivation of Med17 had a severe impact on Pol II association with the test gene induced through artificial tethering of TFIIB, indicating that Mediator can also impact the formation of the PIC in the absence of activator in vivo. Although, a large body of literature supports the view that the interaction with activators serves primarily to recruit Mediator, structural studies showing conformational changes in Mediator in contact with some activators suggest that the latter might also alter qualitatively Mediator in a manner that facilitates its interaction with Pol II/GTFs and drives assembly of the PIC forward $[13,20,43,44]$. Thus the question is now whether Mediator will affect the assembly/stabilization of the PIC in a similar manner in the presence and in the absence of activator.

In this study, we further exploited our activator-independent in vivo transcription assay to investigate the mechanism of action of Mediator in the assembly of the Pol II transcription initiation machinery in the absence of activator in vivo. Our results, using ChIP and sequential-ChIP in combination with existing ts mutants of Kin28 and Med17 as well as new ts mutants of Med14 $\mathrm{N}$-terminal half, provide evidence that in vivo (i) Mediator can be recruited to a promoter as a CDK-free complex in the absence of contact with an activator, and (ii) Mediator can impact the overall assembly/stability of the transcription initiation machinery without input/signals from an activator. 


\section{Materials and Methods}

\subsection{Yeast strains, plasmids and media}

Strains used in this study (Table S1) were generated using methods for one-step integration of PCR fragments combined to classical genetic manipulations including diploid construction, sporulation and tetrad dissection. Y667 was constructed by transferring the med14::KanMX4 allele from EUROSCARF strain Y22686 (BY4743; YLR071c/YLR071c::kanMX4) into YPH499 transformed with pVV208-MED14. The met17:: $P_{T E F}-b l e-T_{T E F}-X M E T 17-G F P$ gene was constructed by integrating at the MET17 locus between positions -448 and +1251 (relative to MET17 start codon) a PCR fragment obtained by amplification of plasmid pRS306-P $P_{T E F}-b l e-T_{T E F}-X M E T 17-G F P$ with primers 5'ATGCTATAATAGACATTTAAATCCAATTCTTCCAACATACAATGGGAGCAGCTGAAGCT TCGTACGC3' and

\section{5'TGGAAGTCTGCAATAATGTCATCAATAAATTCGATACCAACAGAGCTCGAGGTCGACG}

GTATCGAT3' (the underlines indicate the sequence of MET17 from -494 to -448 and +1251 to +1295 , respectively).

Plasmid pRS306-P $P_{\text {TEF- }}$ ble- $T_{\text {TEF- }}$ XMET17-GFP was obtained by subcloning into $\mathrm{pRS306}$, first a EcoRI-HindIII fragment from YCplac33-xMET17-GFP [40] containing xMET17 followed by GFP coding sequence and CYC1 terminator, and second a NotI fragment from pUG66 containing the ble coding sequence flanked by Ashbya gossypii TEF promoter and terminator. xMET17 consists in MET17 from positions -1 to -400 with the sequence CAGTTGCCTAGCAACTACATATGGTCACC including the 13-mer X-box of the polyomavirus enhancer (underlined, with the two inverted repeats in italic) at position -200. Plasmids pRS313-, pRS314-, and pRS313-TFIIB-RFX used here were derived from pRS313-TFIIB-RFX used in Lacombe et al [40] by deleting the HA epitope at the junction of TFIIB and RFX as well as the first 90 amino acids of RFX. Plasmid pRS315-MaxRFX (a gift from Michel Strubin) contains a chimeric gene, expressed under the control of SPT15 promoter and terminator regions, consisting in SV40 nuclear localization signal followed by the 72-amino acid long helix-loop-helix motif of the human Max protein fused to amino acid 92 of RFX [45]. Plasmids pVV204-MED14 and pVV208-MED14 (kindly provided by Julie Soutourina, CEA/Saclay) consist of MED14 coding 
sequence cloned into pVV204 (TRP1, CEN) and pVV208 (URA3, CEN) using the Gateway ${ }^{\mathrm{TM}}$ recombinatorial cloning method.

YPD medium contains $1 \%$ yeast extract, 2\% bacto-peptone and $2 \%$ glucose. YNB medium contains $0.7 \%$ yeast nitrogen base, $0.5 \%$ ammonium sulfate and $2 \%$ glucose. CSM medium contains a CSM amino acid drop-out mixture in addition to YNB. B medium is a synthetic medium lacking organic and inorganic sulfur sources [46].

\subsection{Construction of the med14 temperature sensitive mutants}

Mutants med14-ts9 and med14-ts11 were generated as described in Soutourina et al [14] by in vitro mutagenesis using error-prone PCR followed by screening for ts alleles at $37^{\circ} \mathrm{C}$. MED14 was amplified from pVV208-MED14 with a forward primer starting at position +576 within MED14 coding sequence and a reverse primer starting at position +1780 . PCR was performed using Taq DNA polymerase (New England Biolabs) in the presence of $5 \mathrm{mM} \mathrm{MgCl} 2$ and $0.25 \mathrm{mM} \mathrm{MnCl}_{2}$. The PCR product was co-transformed into strain Y667 alongside with an engineered version of pVV204-MED14 containing two unique BspE1 restriction sites within MED14 at positions +664 and +1561 , which was purified after digestion by BspE1. The pVV208-MED14 plasmid was counter-selected on 5-FOA medium and colonies were then replicated in duplicates on YPD plates and incubated at $30^{\circ} \mathrm{C}$ or $37^{\circ} \mathrm{C}$ for three days to identify ts clones. Plasmids were isolated from the ts clones, sequenced, and re-transformed into Y667 to confirm the phenotypes.

\subsection{Chromatin immunoprecipitation (ChIP) protocol}

Cell fixation, chromatin preparation and immunoprecipitation were performed essentially as described previously $[30,40]$ with several modifications. In short: formaldehyde-fixed cells were disrupted using a FastPrep instrument; the crosslinked chromatin was collected by centrifugation for 60 $\min$ at $20,000 \times g$; the pellet was first solubilized by sonicating with a Vibra-Cell ultrasonic processor (500 Watt model equipped with a 3-mm stepped microtip, Sonics and Materials, Inc.) set at amplitude $25 \%$ (6 times $10 \mathrm{sec}$ at $4^{\circ} \mathrm{C}$ ), and then the crosslinked chromatin was further sheared for $500 \mathrm{sec}$ at $4^{\circ} \mathrm{C}$ in a S220 focused-ultrasonicator (Covaris) with peak incident power set at $140 \mathrm{~W}$ and duty cycle at $12 \%$, 
to generate DNA fragments ranging from 100 to $400 \mathrm{bp}$ with an average of $200 \mathrm{bp}$; finally the remaining debris was eliminated by centrifugation at $10,000 \mathrm{x} g$ for $15 \mathrm{~min}$.

Antibodies used include: anti-Rpb1 rabbit polyclonal antibody y-80, anti-RFX1 rabbit polyclonal antibody H-230X, and anti-TBP rabbit polyclonal antibody y-240 (all from Santa Cruz Biotechnology). Typically, immunoprecipitation was performed by incubating overnight at $4^{\circ} \mathrm{C}$ crosslinked chromatin extracted from $5 \times 10^{7}$ cells with $2 \mu \mathrm{g}$ (anti-Rpb1 and anti-TBP) or $8 \mu \mathrm{g}$ (anti-RFX1) of antibodies in a final volume of $400 \mu \mathrm{L}$. TAP-tagged proteins were immunoprecipitated by incubating the same amount of crosslinked chromatin with $30 \mu \mathrm{l}$ of rabbit IgG-agarose (Sigma) for 4 hours at $4^{\circ} \mathrm{C}$. After reverse-crosslinking and purification, DNA was quantified by real time PCR using the LightCycler 480 instrument (Roche). Sequences of primers are given in Table S2. A typical run included duplicates or triplicates for each immunoprecipitated (IP) and input DNA, and serial dilutions of one input (Total) DNA to create a standard curve and to determine the efficiency of the amplification. Data was analyzed with the LightCycler 480 software using the "second derivative maximum" method for quantification. For each set of immunoprecipitation experiments, occupancy of the immunoprecipitated protein at the gene position determined by the primer pair was calculated by dividing the qPCR quantification value for the IP by the qPCR quantification value for the Total, giving the IP/Total fraction, and then by normalizing with the IP/Total number obtained for IME2 in the wild type strain included in the set.

\subsection{Sequential Chromatin immunoprecipitation (Sequential-ChIP) protocol}

Cell fixation with formaldehyde and chromatin preparation was performed as described above except that chromatin was sheared using the Vibra-Cell ultrasonic processor only, 8 times $20 \sec$ at $4^{\circ} \mathrm{C}$ with amplitude control set at $40 \%$, to generate DNA fragments ranging from 100 to $1,000 \mathrm{bp}$ with an average of $500 \mathrm{bp}$.

Chromatin extract from $10^{8}$ cells diluted in $400 \mu \mathrm{L}$ of FA-lysis buffer $(50 \mathrm{mM}$ Hepes-KOH, pH7.5, $150 \mathrm{mM} \mathrm{NaCl}, 1 \mathrm{mM}$ EDTA, $1 \%$ Triton X-100, 0.1\% sodium deoxycholate, $0.1 \%$ SDS) was incubated for 7 hours at $4{ }^{\circ} \mathrm{C}$ on a rotating wheel with $25 \mu \mathrm{L}$ of rabbit IgG-agarose beads blocked with $500 \mu \mathrm{g}$ of acetylated-BSA. Beads were collected by centrifugation at $1000 \mathrm{x} g$ for $1 \mathrm{~min}$ and washed four times with FA-lysis buffer. Immunoprecipitated chromatin was eluted by heating for $10 \mathrm{~min}$ at 
$45^{\circ} \mathrm{C}$ in $125 \mu \mathrm{L}$ of elution buffer ( $25 \mathrm{mM}$ Tris- $\mathrm{HCl} \mathrm{pH} 7.5,5 \mathrm{mM}$ EDTA, $1 \%$ SDS), and IgG-agarose beads were eliminated by centrifugation through a $0.22 \mu \mathrm{m}$ cellulose acetate filter (Spin-X centrifuge tube filter; Costar). $25 \mu \mathrm{L}$ of eluate was removed for subsequent analysis of the first immunoprecipitation. The remaining $100 \mu \mathrm{L}$ was diluted with $900 \mu \mathrm{L}$ of FA-lysis buffer without SDS and $500 \mu \mathrm{g}$ of acetylated-BSA was added. The second immunoprecipitation was performed overnight in the presence (or not in the case of the "mock") of $2 \mu \mathrm{g}$ of anti-Rpb1 antibodies (y-80, Santa Cruz Biotechnology). For comparison, an aliquot of the starting chromatin extract (one twentieth) was diluted in $1 \mathrm{~mL}$ of FA lysis buffer and immunoprecipitated in parallel. Immunocomplexes were captured by incubating for 4 hours at $4^{\circ} \mathrm{C}$ on a rotating wheel with $20 \mu \mathrm{L}$ of protein A-Sepharose blocked with formaldehyde-crosslinked and sheared chromatin from Kluyveromyces lactis. Beads were washed, immunoprecipitated chromatin was eluted, and formaldehyde crosslinking was reversed as in the ChIP protocol. DNA was also purified and quantified as in the ChIP protocol. Sequences of primers are given in Table S2. Occupancy was calculated as in the ChIP protocol. Co-occupancy was calculated by dividing the qPCR quantification value for the second (anti-Rpb1) IP by the qPCR quantification value for the first (anti-TAP) IP, and then by normalizing with the IP/Total value for IME2 in the wild type obtained in the immunoprecipitation experiment done in parallel in which the extracts were incubated directly with the anti-Rpb1 antibody.

\subsection{RNA analysis}

RNA extraction and reverse transcription (RT)-quantitative PCR were conducted as described in Lacombe et al [40]. Sequence of primers is given in Table S3. 


\section{Results}

\subsection{Experimental in vivo transcription system activator-independent}

In this study we used an upgraded version of the system in which Pol II is brought to a test promoter through a physical connection between TFIIB and the human DNA-binding protein RFX $[40,47]$. Binding to the test promoter is ensured by the presence of the 13-mer RFX DNA-binding site of the polyomavirus enhancer 72 base pairs upstream of the TATA box of the reporter gene consisting in the MET17 promoter fused to the coding sequence of GFP (xMET17-GFP). We showed previously that binding of TFIIB-RFX to $x M E T 17$ allowed robust Pol II recruitment in the absence of activator as well as transcription initiation at the normal start sites [40]. To avoid potential biases resulting from variations in copy number, the $x M E T 17-G F P$ reporter was in this work integrated into the chromosome (see Materials and Methods). As in our previous report [40], Pol II is strongly recruited to the reporter gene in the presence of TFIIB-RFX, but not in the presence of TFIIB alone or in combination with MaxRFX (which consists in RFX fused to a 72-amino acid fragment containing the helix-loop-helix domain of the mammalian oncoprotein Max [45]) (Fig. S1A, upper graphs). In addition, Pol II can be found at the beginning as well as at the end of the coding sequence of the reporter (Fig. S1A, upper graphs), demonstrating efficient elongation, as confirmed by the accumulation of mRNAs (Fig. S1B). All eight housekeeping genes analyzed as controls are transcribed at similar levels in the presence of TFIIB and TFIIB-RFX (Fig. S1B), indicating that the fusion of TFIIB to RFX does not affect transcription globally. Note that $X M E T 17$ contains the binding site for the activator Met4 but, under our growth conditions of excess methionine, Met4 is degraded through the ubiquitin-proteasome system and cannot bind and activate $M E T 17[30,40,48]$.

\subsection{Activator-independent recruitment of CDK-free Mediator}

We previously published that recruitment of the Pol II transcription machinery through TFIIBRFX is accompanied by the recruitment of Mediator [40]. In two subsequent studies by others, a genome-wide increase in Mediator occupancy at the core promoter was observed upon inactivation or depletion of the Kin28 subunit of TFIIH, highlighting the specific role of Kin28 in the dynamics of association of Mediator with promoters [29,35]. To gain additional insight into the underlying 
mechanisms, we analyzed Mediator recruitment to $x M E T 17-G F P$ in the presence of the kin28-ts3 mutation which causes at $37^{\circ} \mathrm{C}$ a global decrease in mRNA synthesis [21] as well as a remarkable decrease in Pol II association with $x M E T 17-G F P$ (Fig. S1A, lower graphs). To monitor Mediator recruitment, we first used strains expressing TAP-tagged version of Med14 and Med17. The wild type and kin28-ts3 TAP-tagged strains expressing TFIIB-RFX show at permissive temperature $\left(26^{\circ} \mathrm{C}\right)$ similar levels of Pol II association with $x$ MET17-GFP (Fig. S2A), resulting in similar transcription levels (Fig. S2B). As observed in our previous study [40], incubation at $37^{\circ} \mathrm{C}$ lead to a sharp decrease (4- to 6-fold) in Pol II association with $x M E T 17-G F P$ coding sequence in the mutants compared to the wild type strains (Fig. 1, upper graphs). Pol II association with PMA1 coding sequence is also strongly reduced, contrasting with studies using chemical inactivation $[49,50]$ or conditional depletion from the nucleus [35], in which Pol II association with coding sequences was reported to be only slightly decreased, in a gradual manner toward the 3' ends, upon Kin28 inactivation/depletion. This discrepancy is certainly due to the fact that the kin28-ts3 mutation destabilizes the TFIIH complex at restrictive temperature, causing dissociation of Rad3 as well [51], hence a stronger effect on the stability of the PIC.

In contrast to Pol II, Med14 and Med17 association with the xMET17-GFP promoter in the presence of TFIIB-RFX is increased by three fold in the TAP-tagged kin28-ts3 mutants compared to the wild type strains (Fig. 1A and 1B, lower graphs). Expressing RFX disconnected from TFIIB does not lead to Mediator recruitment (Fig. 1A, IIB + MaxRFX). As an additional control we also measured Pol II and Mediator association with MET2 which belongs to the same network as MET17 and is not activated in the methionine-rich growth conditions of the experiment; as expected, no significant association is observed (Fig. 1A and B). Importantly, no enrichment of $x M E T 17-G F P$ is observed in anti-TAP immunoprecipitates from untagged chromatin extracts (Fig. S3A; see also Fig. 3), and moreover Med14-TAP association is restricted to the promoter region of $x M E T 17-G F P$ and does not extend over the entire gene body (Fig. S3B), together arguing against artefactual ChIP signals that would be due to increased transcription as reported by Teytelman et al. [52]. We also used KIN28 and kin28ts3 strains expressing a TAP-tagged CDK8 (Fig. 1C): in sharp contrast with Med14-TAP and Med17TAP, CDK8-TAP occupancy at XMET17 was very low (maximum 2-fold over the background level), and no higher occupancy was observed in the kin28-ts3 mutant, but instead slightly lower occupancy. 
Altogether these results confirm that Mediator can be recruited to a transcriptionally active promoter in the absence of contact with an activator, and they provide evidence that the form of Mediator that is recruited in this situation does not include the CDK module.

\subsection{Interplay between Mediator and Pol II recruitment}

The fact that Pol II occupancy at $x M E T 17$ was decreased in kin28-ts3 at $37^{\circ} \mathrm{C}$ while at the same time Mediator occupancy was increased may seem counterintuitive in the hypothesis that Mediator associates with $x$ MET17 through Pol II and the PIC. However, this could simply be due to Mediator being crosslinked through the small fraction of Pol II that remained present at $x M E T 17$, since Pol II association was diminished in kin28-ts3 but not abolished. To test this hypothesis, we performed cooccupancy analysis by doing sequential ChIP of Med14-TAP followed by Rpb1 (see Materials and Methods). To maximize the amount of Pol II associated and crosslinked to promoters, chromatin extracts were prepared from cells exposed to restrictive temperature for $30 \mathrm{~min}$ instead of $45 \mathrm{~min}$ as in the experiments above. The results (Fig. 2) showed that: (i) the xMET17 fragment from the first immunoprecipitation was re-immunoprecipitated by the anti-Rpb1 antibodies 10 - to 15 -fold more efficiently than the IME2 negative-control fragment, and 2- to 3-fold more efficiently than the PMA1 upstream activating sequence (UAS) fragment, where Mediator is primarily bound through the activator

(Fig. 2B, Med14-Tap + Rpb1); (ii) no addition of anti-Rpb1 antibody into the second immunoprecipitation (Fig. 2B, Med14-TAP + mock) led to no additional enrichment of $x M E T 17$ over IME2.

The results show that $x M E T 17$ was co-occupied by Med14 and Pol II in both the wild type and the kin28-ts3 mutant (Fig. 2C). Importantly, the proportion of $x M E T 17$ fragment containing Rpb1 at the same time as Med14 is not lower in kin28-ts3 than in the wild type but slightly higher, thus confirming the hypothesis that Mediator was crosslinked to XMET17 in kin28-ts3 through the small fraction of Pol II that remained present, and also affirming that crosslinking of Mediator to $x M E T 17$ is strictly dependent on the presence of Pol II.

\subsection{Effect of inactivation of Mediator Head module on assembly of Pol II transcription machinery}

Together with our published work, the results above reveal Mediator's capability to stabilize Pol II association with the promoter independently of activators; however they do not give information about 
how the GTFs forming the PIC alongside Pol II are affected. To address this aspect we used the med17138 mutant to assess by ChIP the effect of Med17 inactivation on the recruitment to XMET17 of several GTFs, including the Kin28 subunit of TFIIH, the TFA2 subunit of TFIIE, the TATA-binding protein TBP, and TFIIB. To immunoprecipitate Kin28 and TFA2, TAP-tagged versions of the proteins were constructed (see Materials and Methods). TBP was immunoprecipitated with anti-TBP antibodies and TFIIB with antibodies against the RFX moiety of TFIIB-RFX. Transcriptional analysis showed that at permissive temperature $\left(26^{\circ} \mathrm{C}\right) \times M E T 17-G F P$ was induced by TFIIB-RFX at similar levels in all strains (Fig. S4). At restrictive temperature $\left(37^{\circ} \mathrm{C}\right)$, association of Rpb1 with $x M E T 17-G F P$ was 7 -fold (Kin28-TAP strain) to 8-fold (TFA2-TAP strain) lower in med17-138 than in the wild type (Fig. 3A). At the same time, association of the four GTFs with $x M E T 17-G F P$ was also significantly diminished in med17-138: by 4.2-fold for Kin28-TAP, 6.5-fold for TFA2-TAP, 3.5-fold for TBP and 4.3-fold for TFIIB (Fig. 3A, lower graph, and Fig. 3B). Noteworthy, the $x M E T 17$ promoter showed in the wild type cells a threefold higher TFIIB-RFX occupancy compared to the PMA1 promoter (Fig. 3B, graph on the right), while occupancy of the two promoters by Rpb1, TBP, TFA2 and Kin28, was similar; furthermore, in the mutant at $37^{\circ} \mathrm{C}$, TFIIB-RFX occupancy at $x M E T 17$ remained high compared to PMA1, which was at the background level (Fig. 3B, graph on the right). These results likely reflects the fact that: (i) TFIIB-RFX can be crosslinked to DNA through both TFIIB and RFX at $x M E T 17$, but only through TFIIB at PMA1; and/or (ii) RFX association with its DNA-binding site is maintained after Med17 inactivation while TFIIB association with the core promoter is disrupted.

Together these results suggest that, when transcription is driven by TFIIB-RFX, the Mediator Head domain has a strong stabilizing effect on the assembly at $x M E T 17$ of the entire Pol II machinery.

\subsection{Effect of inactivation of Mediator Middle module on assembly of Pol II transcription machinery}

We next used two ts mutants in Med14, named med14-ts9 and med14-ts11, which were constructed using an error-prone PCR strategy (see Materials and Methods). These mutants contain respectively 7 substitutions (R293K, N333I, I352T, L389Q, I471S, N495D, and G522W) and 8 substitutions (Y269H, K408I, L438H, W442R, V455A, V498A, I517V, and A537T). They have a strong ts phenotype and display widespread transcriptional defects at non permissive temperature, as judged 
by our transcriptional analyses showing an mRNA decrease at $37^{\circ} \mathrm{C}$ of all 9 housekeeping genes (Fig. 4A and B). As expected transcription of $x M E T 17-G F P$ was induced by the TFIIB-RFX fusion in this genetic background (Fig. S5). To analyze the effect of med14-ts9 and med14-ts11 on Pol II recruitment and PIC assembly, ChIP experiments were performed on cells incubated at restrictive temperature. Pol II was analyzed first: the results showed a severe decrease, after 100 min at $37^{\circ} \mathrm{C}$, of $\mathrm{Rpb} 1$ association with $x M E T 17-G F P$, as well as with the housekeeping genes ADH1, PMA1, TPI1 and PDA1 (by a factor ranging from 5 for $x M E T 17-G F P$ to 17 for PMA1; Fig. 4C). Kin28 and TBP recruitment was analyzed using a strain expressing HA-tagged Kin28 (Fig. 5). Rpb1 recruitment was also measured in this strain to allow direct comparison. The results showed a modest but significant decrease in Kin28-HA and TBP association with $x M E T 17-G F P$ in the two mutants at $37^{\circ} \mathrm{C}$ compared to the wild type: Kin28-HA occupancy was around 2.5-fold lower and TBP occupancy around twofold lower (Fig. 5). Association of both factors with the housekeeping gene PMA1 was also decreased (Fig. 5).

These results indicate that inactivation of Med14 affects Mediator's intrinsic capability to stabilize the assembly of the Pol II machinery even when transcription is not driven by an activator. 


\section{Discussion}

\subsection{Mechanisms of recruitment of Mediator to promoters}

This work provides new insights regarding Mediator's capability to function in the absence of activator input in vivo. Our ChIP results with the kin28-ts3 strain showing increased association of Mediator with the test gene at restrictive temperature, along with the sequential-ChIP results showing co-occupancy with Pol II, provide novel strong evidence that Mediator can be recruited to a promoter even when assembly of the Pol II initiation machinery is driven by artificial anchoring of a GTF in lieu of a classical activator. And the sequential-ChIP results, showing comparable levels of co-occupancy at the $x M E T 17$ promoter in the wild type and kin28-ts3 strains at restrictive temperature, strongly support the conclusion that Mediator association with $x M E T 17$ requires concomitant association of the Pol II machinery. In addition, the Mediator complex that is recruited to xMET17 when transcription is driven through TFIIB-RFX is devoid of CDK8. Therefore, in complement to the two recent studies showing that Mediator is first recruited by enhancer-bound activators as a form containing the CDK module, and this module dissociates before contacts are made with Pol II/GTFs at promoters [41,42], our results suggest an alternative possibility in which the CDK-free form of Mediator would be directly recruited to some promoters in an activator-independent manner, that is with no obligatory contact with activators potentially bound to the UAS.

It was proposed following the finding that Middle/Head subunits are still present at a number of genes in the med3 med15 double-null mutant devoid of the Tail module [42,53], that Mediator might be recruited to promoters in vivo through two distinct mechanisms, one involving interactions with enhancer-bound activator proteins, the other involving interactions with the Pol II initiation machinery at the core promoter. Although this finding in med3 med15 could be explained by the possibility that some yeast activators might interact with subunits outside the Tail, in the same way as in metazoans, our results here and in conjunction with Lacombe et al. [40] demonstrate that the complete core Mediator complex can indeed integrate the PIC without prior interaction with/recruitment by activators. A dual mode of recruitment, activator-dependent or activator-independent, by implying differential dynamics of association and formaldehyde-crosslinking pathways, would also be consistent with the ChIP-based 
genome-wide occupancy studies showing poor correlation between Mediator association levels and transcription activity, and would explain why Mediator is not detected by ChIP at some highly transcribed genes, in spite of being required for their transcription [31,54]. What determinants discriminate between the two mechanisms of recruitment remains to be established. One simple explanation would consider that not all activators are able (and/or need) to interact with Mediator. Following this idea, one can speculate that the capability to interact with Mediator represents a regulatory characteristic of only particular categories of activators, including for instance those mediating fast response to environmental challenges [28,30,55-57]. Activators that are unable would exert their action by recruiting/contacting other players involved in the assembly of the PIC, such as chromatin remodelers/modifiers or TAFs, and in these instances Mediator would be recruited directly through interactions with components of the PIC. It cannot be excluded that additional factors, or specific configurations or conformations, may be required for the association of Mediator with the PIC in the absence of activator contact.

Our results with the kin28-ts3 mutant are consistent with the idea that the association of Mediator with Pol II is released through phosphorylation of the CTD by Kin28 [19,29,35]. They also provide evidence that this event does not obligatorily need input from an activator, in line with earlier results showing that Mediator stimulates CTD phosphorylation by TFIIH in the absence of activator in assays using purified components [4,39]. However, the kin28-ts3 allele causes the loss of Kin28 as well as Rad3 [53]. Henceforth, the effects observed on the overall stability of the PIC in the kin28-ts3 strain at restrictive temperature are not attributable to the loss of Kin28 kinase activity alone.

\subsection{Function of Mediator in the assembly of the Pol II initiation machinery}

Structural studies have suggested that association of Mediator with Pol II, to form the so-called Mediator-Pol II holoenzyme, is accompanied by a significant rearrangement of Mediator conformation, including a change in the relative orientation of the Tail and Middle modules $[15,18,20]$. It is already known from the early experiments that the holoenzyme complex can be formed in reconstituted transcription assays lacking a transcriptional activator [4], implying that Mediator should be able to switch spontaneously between conformations in the absence of regulatory signals in vitro. Assuming 
that Mediator does exist in the cell as a free complex adopting the type of conformations observed in purified preparations, our results suggest that Mediator is also able, at physiological protein concentrations and in the complex environment of the nucleus, to shift from the free conformation to the holoenzyme conformation in the absence of activator input. Several studies have already used ts mutations within various Middle and Head module subunits, including Med10, Med11 and Med 17, to investigate genome-wide how the yeast Mediator affects the assembly at promoters of individual GTFs in addition to Pol II $[11,32,33]$. The results of these studies highlight the importance of Mediator in the formation of the PIC; however, they do not give information on whether, or not, Mediator needs to be interacting with an activator in order to exert its function in the recruitment of GTFs/Pol II. Our results here presented provide evidence that Mediator mutations affect the overall stability of the PIC also in a context where no activator is involved. We show that med17-138 at restrictive temperature causes a parallel decrease in association with the xMET17 reporter of all Pol II, TBP, TFIIB, TFA2 (TFIIE) and Kin28 (TFIIH), suggesting destabilization of the entire PIC upon inactivation of Med17 even when transcription is not driven by an activator. At molecular level, this could be a result either from a single disruption of a key interaction with one specific GTF essential for the nucleation of the PIC, for example TBP, which binds Mediator Head module via a subcomplex, the Med8-Med18-Med20 triad [12], that is lost in med17-138 at restrictive temperature $[22,23]$ or, alternatively, a simultaneous loss of multiple contacts with several components of the PIC since the med17-138 allele was shown to strongly destabilize the Mediator complex at restrictive temperature, causing partial dislocation [22] (see also Fig. S5).

In our study we also observed destabilization of the PIC in the med14-ts9 and med14-ts11 mutants. Recent structural data $[15,18]$ indicated that the N-terminal of Med14 is located in Mediator Middle module and it establishes contacts with Med17. Henceforth, it is likely that the mutations in med14-ts9 and med14-ts11, given their positions in the second quarter of Med14 polypeptide chain, affect the interactions among the middle module subunits and the connection between the Middle and Head modules; thus, subsequently disrupting the stability of the Head module.

\subsection{Conclusion}


As a whole our findings are in agreement with the existing evidence that Mediator would act by enhancing the rate of formation and/or the stability of the PIC and, in addition to other studies, they provide the proof-of-principle that the capacity of Mediator to act on the PIC in vivo does not necessarily requires a contact with an activator, supporting the model that Mediator might function not always as a co-activator "sensu stricto", but also independently of activators. 


\section{Acknowledgements}

We thank Michel Strubin (University of Geneva) and Julie Soutourina (CEA/Saclay) for plasmids and strains. We are grateful to Julien Chaillot for constructing the plasmid pRS306-ble ${ }^{r}$-xMET17-GFP and

to Lisete Fernandes for her comments on the manuscript. This work was supported by Centre National de la Recherche Scientifique and Agence Nationale pour la Recherche [ANR-08-BLAN-0229]. 


\section{References}

[1] B.L. Allen, D.J. Taatjes, The Mediator complex: a central integrator of transcription, Nat. Rev. Mol. Cell Biol. 16 (2015) 155-166.

[2] C. Jeronimo, F. Robert, The Mediator Complex: At the Nexus of RNA Polymerase II Transcription, Trends Cell Biol. 27 (2017) 765-783.

[3] H.-M. Bourbon, Comparative genomics supports a deep evolutionary origin for the large, fourmodule transcriptional mediator complex, Nucleic Acids Res. 36 (2008) 3993-4008.

[4] Y.J. Kim, S. Björklund, Y. Li, M.H. Sayre, R.D. Kornberg, A multiprotein mediator of transcriptional activation and its interaction with the C-terminal repeat domain of RNA polymerase II, Cell. 77 (1994) 599-608.

[5] S.A. Ansari, R.H. Morse, Mechanisms of Mediator complex action in transcriptional activation, Cell. Mol. Life Sci. CMLS. (2013).

[6] T. Borggrefe, X. Yue, Interactions between subunits of the Mediator complex with gene-specific transcription factors, Semin. Cell Dev. Biol. 22 (2011) 759-768.

[7] S. Malik, R.G. Roeder, The metazoan Mediator co-activator complex as an integrative hub for transcriptional regulation, Nat. Rev. Genet. 11 (2010) 761-772.

[8] F.J. Asturias, Y.W. Jiang, L.C. Myers, C.M. Gustafsson, R.D. Kornberg, Conserved structures of mediator and RNA polymerase II holoenzyme, Science. 283 (1999) 985-987.

[9] M.R. Dotson, C.X. Yuan, R.G. Roeder, L.C. Myers, C.M. Gustafsson, Y.W. Jiang, Y. Li, R.D. Kornberg, F.J. Asturias, Structural organization of yeast and mammalian mediator complexes, Proc. Natl. Acad. Sci. U. S. A. 97 (2000) 14307-14310.

[10] T. Borggrefe, R. Davis, H. Erdjument-Bromage, P. Tempst, R.D. Kornberg, A complex of the Srb8, $-9,-10$, and -11 transcriptional regulatory proteins from yeast, J. Biol. Chem. 277 (2002) $44202-44207$.

[11] C. Esnault, Y. Ghavi-Helm, S. Brun, J. Soutourina, N. Van Berkum, C. Boschiero, F. Holstege, M. Werner, Mediator-dependent recruitment of TFIIH modules in preinitiation complex, Mol. Cell. 31 (2008) 337-346.

[12] L. Larivière, S. Geiger, S. Hoeppner, S. Röther, K. Strässer, P. Cramer, Structure and TBP binding of the Mediator head subcomplex Med8-Med18-Med20, Nat. Struct. Mol. Biol. 13 (2006) 895-901.

[13] P.J. Robinson, M.J. Trnka, D.A. Bushnell, R.E. Davis, P.-J. Mattei, A.L. Burlingame, R.D. Kornberg, Structure of a Complete Mediator-RNA Polymerase II Pre-Initiation Complex, Cell. 166 (2016) 1411-1422.e16.

[14] J. Soutourina, S. Wydau, Y. Ambroise, C. Boschiero, M. Werner, Direct interaction of RNA polymerase II and mediator required for transcription in vivo, Science. 331 (2011) 1451-1454.

[15] K.-L. Tsai, X. Yu, S. Gopalan, T.-C. Chao, Y. Zhang, L. Florens, M.P. Washburn, K. Murakami, R.C. Conaway, J.W. Conaway, F.J. Asturias, Mediator structure and rearrangements required for holoenzyme formation, Nature. 544 (2017) 196-201.

[16] T. Imasaki, G. Calero, G. Cai, K.-L. Tsai, K. Yamada, F. Cardelli, H. Erdjument-Bromage, P. Tempst, I. Berger, G.L. Kornberg, F.J. Asturias, R.D. Kornberg, Y. Takagi, Architecture of the Mediator head module, Nature. 475 (2011) 240-243.

[17] L. Larivière, C. Plaschka, M. Seizl, L. Wenzeck, F. Kurth, P. Cramer, Structure of the Mediator head module, Nature. 492 (2012) 448-451.

[18] P.J. Robinson, M.J. Trnka, R. Pellarin, C.H. Greenberg, D.A. Bushnell, R. Davis, A.L. Burlingame, A. Sali, R.D. Kornberg, Molecular architecture of the yeast Mediator complex, ELife. 4 (2015). http://elifesciences.org/lookup/doi/10.7554/eLife.08719 (accessed August 2, 2017).

[19] P.J.J. Robinson, D.A. Bushnell, M.J. Trnka, A.L. Burlingame, R.D. Kornberg, Structure of the Mediator Head module bound to the carboxy-terminal domain of RNA polymerase II, Proc. Natl. Acad. Sci. 109 (2012) 17931-17935.

[20] K.-L. Tsai, C. Tomomori-Sato, S. Sato, R.C. Conaway, J.W. Conaway, F.J. Asturias, Subunit Architecture and Functional Modular Rearrangements of the Transcriptional Mediator Complex, Cell. 157 (2014) 1430-1444. 
[21] F.C. Holstege, E.G. Jennings, J.J. Wyrick, T.I. Lee, C.J. Hengartner, M.R. Green, T.R. Golub, E.S. Lander, R.A. Young, Dissecting the regulatory circuitry of a eukaryotic genome, Cell. 95 (1998) 717-728.

[22] T. Linder, X. Zhu, V. Baraznenok, C.M. Gustafsson, The classical srb4-138 mutant allele causes dissociation of yeast Mediator, Biochem. Biophys. Res. Commun. 349 (2006) 948-953.

[23] Y. Takagi, R.D. Kornberg, Mediator as a general transcription factor, J. Biol. Chem. 281 (2006) 80-89.

[24] N. Petrenko, Y. Jin, K.H. Wong, K. Struhl, Evidence that Mediator is essential for Pol II transcription, but is not a required component of the preinitiation complex in vivo, ELife. 6 (2017).

[25] L. Warfield, S. Ramachandran, T. Baptista, D. Devys, L. Tora, S. Hahn, Transcription of Nearly All Yeast RNA Polymerase II-Transcribed Genes Is Dependent on Transcription Factor TFIID, Mol. Cell. 68 (2017) 118-129.e5.

[26] L.T. Bhoite, Y. Yu, D.J. Stillman, The Swi5 activator recruits the Mediator complex to the HO promoter without RNA polymerase II, Genes Dev. 15 (2001) 2457-2469.

[27] G.O. Bryant, M. Ptashne, Independent recruitment in vivo by Gal4 of two complexes required for transcription, Mol. Cell. 11 (2003) 1301-1309.

[28] L. Kuras, T. Borggrefe, R.D. Kornberg, Association of the Mediator complex with enhancers of active genes, Proc. Natl. Acad. Sci. U. S. A. 100 (2003) 13887-13891.

[29] C. Jeronimo, F. Robert, Kin28 regulates the transient association of Mediator with core promoters, Nat. Struct. Mol. Biol. 21 (2014) 449-455.

[30] C. Leroy, L. Cormier, L. Kuras, Independent recruitment of mediator and SAGA by the activator Met4, Mol. Cell. Biol. 26 (2006) 3149-3163.

[31] E. Paul, Z.I. Zhu, D. Landsman, R.H. Morse, Genome-Wide Association of Mediator and RNA Polymerase II in Wild-Type and Mediator Mutant Yeast, Mol. Cell. Biol. 35 (2015) 331-342.

[32] F. Eyboulet, S. Wydau-Dematteis, T. Eychenne, O. Alibert, H. Neil, C. Boschiero, M.-C. Nevers, H. Volland, D. Cornu, V. Redeker, M. Werner, J. Soutourina, Mediator independently orchestrates multiple steps of preinitiation complex assembly in vivo, Nucleic Acids Res. 43 (2015) 9214-9231.

[33] T. Eychenne, E. Novikova, M.-B. Barrault, O. Alibert, C. Boschiero, N. Peixeiro, D. Cornu, V. Redeker, L. Kuras, P. Nicolas, M. Werner, J. Soutourina, Functional interplay between Mediator and TFIIB in preinitiation complex assembly in relation to promoter architecture, Genes Dev. 30 (2016) 2119-2132.

[34] J.Q. Svejstrup, Y. Li, J. Fellows, A. Gnatt, S. Bjorklund, R.D. Kornberg, Evidence for a mediator cycle at the initiation of transcription, Proc. Natl. Acad. Sci. U. S. A. 94 (1997) 60756078.

[35] K.H. Wong, Y. Jin, K. Struhl, TFIIH Phosphorylation of the Pol II CTD Stimulates Mediator Dissociation from the Preinitiation Complex and Promoter Escape, Mol. Cell. 54 (2014) 601612.

[36] R.C. Conaway, J.W. Conaway, The Mediator complex and transcription elongation, Biochim. Biophys. Acta BBA - Gene Regul. Mech. 1829 (2013) 69-75.

[37] H.J. Baek, Y.K. Kang, R.G. Roeder, Human Mediator enhances basal transcription by facilitating recruitment of transcription factor IIB during preinitiation complex assembly, J. Biol. Chem. 281 (2006) 15172-15181.

[38] G. Mittler, E. Kremmer, H.T. Timmers, M. Meisterernst, Novel critical role of a human Mediator complex for basal RNA polymerase II transcription, EMBO Rep. 2 (2001) 808-813.

[39] L.C. Myers, C.M. Gustafsson, D.A. Bushnell, M. Lui, H. Erdjument-Bromage, P. Tempst, R.D. Kornberg, The Med proteins of yeast and their function through the RNA polymerase II carboxy-terminal domain, Genes Dev. 12 (1998) 45-54.

[40] T. Lacombe, S.L. Poh, R. Barbey, L. Kuras, Mediator is an intrinsic component of the basal RNA polymerase II machinery in vivo, Nucleic Acids Res. 41 (2013) 9651-9662.

[41] N. Petrenko, Y. Jin, K.H. Wong, K. Struhl, Mediator Undergoes a Compositional Change during Transcriptional Activation, Mol. Cell. 64 (2016) 443-454. 
[42] C. Jeronimo, M.-F. Langelier, A.R. Bataille, J.M. Pascal, B.F. Pugh, F. Robert, Tail and Kinase Modules Differently Regulate Core Mediator Recruitment and Function In Vivo, Mol. Cell. 64 (2016) 455-466.

[43] A.M. Naar, Human CRSP interacts with RNA polymerase II CTD and adopts a specific CTDbound conformation, Genes Dev. 16 (2002) 1339-1344.

[44] D.J. Taatjes, A.M. Näär, F. Andel 3rd, E. Nogales, R. Tjian, Structure, function, and activatorinduced conformations of the CRSP coactivator, Science. 295 (2002) 1058-1062.

[45] N. Klages, M. Strubin, Stimulation of RNA polymerase II transcription initiation by recruitment of TBP in vivo, Nature. 374 (1995) 822-823.

[46] L. Cormier, R. Barbey, L. Kuras, Transcriptional plasticity through differential assembly of a multiprotein activation complex, Nucleic Acids Res. 38 (2010) 4998-5014.

[47] E. Gonzalez-Couto, N. Klages, M. Strubin, Synergistic and promoter-selective activation of transcription by recruitment of transcription factors TFIID and TFIIB, Proc. Natl. Acad. Sci. U. S. A. 94 (1997) 8036-8041.

[48] L. Kuras, A. Rouillon, T. Lee, R. Barbey, M. Tyers, D. Thomas, Dual regulation of the met4 transcription factor by ubiquitin-dependent degradation and inhibition of promoter recruitment, Mol. Cell. 10 (2002) 69-80.

[49] J.B. Rodríguez-Molina, S.C. Tseng, S.P. Simonett, J. Taunton, A.Z. Ansari, Engineered Covalent Inactivation of TFIIH-Kinase Reveals an Elongation Checkpoint and Results in Widespread mRNA Stabilization, Mol. Cell. 63 (2016) 433-444.

[50] H. Kim, B. Erickson, W. Luo, D. Seward, J.H. Graber, D.D. Pollock, P.C. Megee, D.L. Bentley, Gene-specific RNA polymerase II phosphorylation and the CTD code, Nat. Struct. Mol. Biol. 17 (2010) 1279-1286.

[51] E.I. Kanin, R.T. Kipp, C. Kung, M. Slattery, A. Viale, S. Hahn, K.M. Shokat, A.Z. Ansari, Chemical inhibition of the TFIIH-associated kinase Cdk7/Kin28 does not impair global mRNA synthesis, Proc. Natl. Acad. Sci. U. S. A. 104 (2007) 5812-5817.

[52] L. Teytelman, D.M. Thurtle, J. Rine, A. van Oudenaarden, Highly expressed loci are vulnerable to misleading ChIP localization of multiple unrelated proteins, Proc. Natl. Acad. Sci. U. S. A. 110 (2013) 18602-18607.

[53] S.A. Ansari, M. Ganapathi, J.J. Benschop, F.C.P. Holstege, J.T. Wade, R.H. Morse, Distinct role of Mediator tail module in regulation of SAGA-dependent, TATA-containing genes in yeast, EMBO J. 31 (2012) 44-57.

[54] X. Fan, K. Struhl, Where does mediator bind in vivo?, PloS One. 4 (2009) e5029.

[55] C.K. Govind, S. Yoon, H. Qiu, S. Govind, A.G. Hinnebusch, Simultaneous recruitment of coactivators by Gen $4 \mathrm{p}$ stimulates multiple steps of transcription in vivo, Mol. Cell. Biol. 25 (2005) 5626-5638.

[56] X. Fan, D.M. Chou, K. Struhl, Activator-specific recruitment of Mediator in vivo, Nat. Struct. Mol. Biol. 13 (2006) 117-120.

[57] S. Kim, D.S. Gross, Mediator recruitment to heat shock genes requires dual Hsf1 activation domains and mediator tail subunits Med15 and Med16, J. Biol. Chem. 288 (2013) 12197-12213. 
A MED14-TAP strain
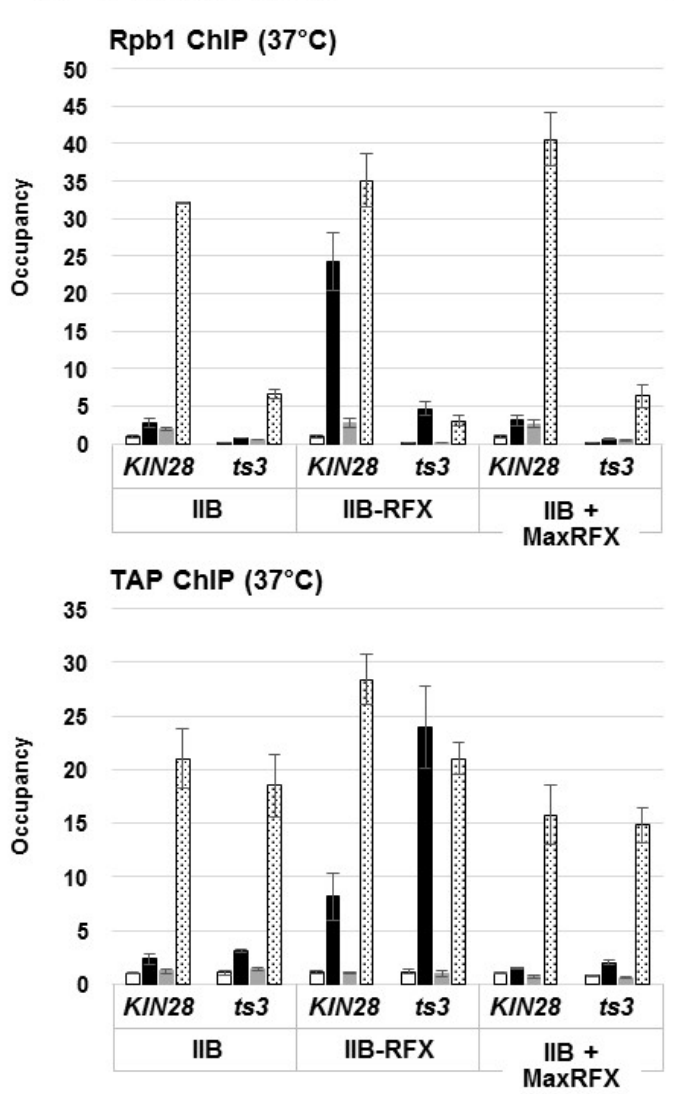

B MED17-TAP strain

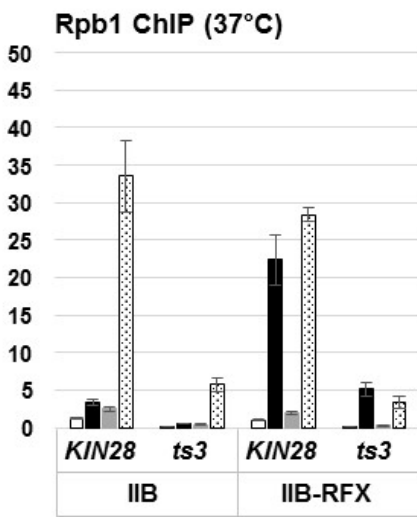

TAP ChIP $\left(37^{\circ} \mathrm{C}\right)$

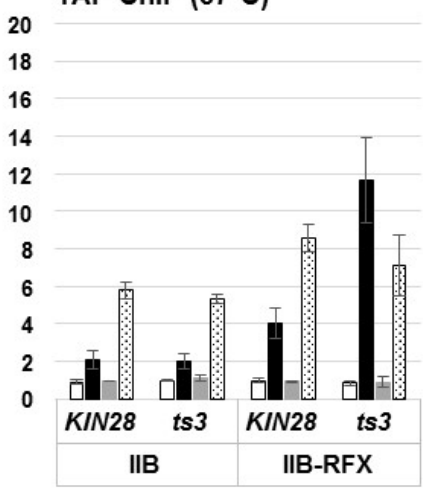

C CDK8-TAP strain

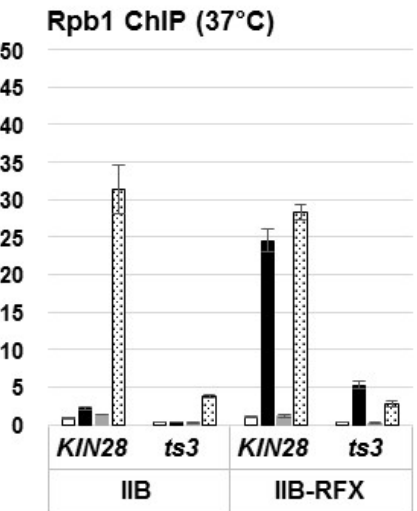

10

TAP ChIP $\left(37^{\circ} \mathrm{C}\right)$

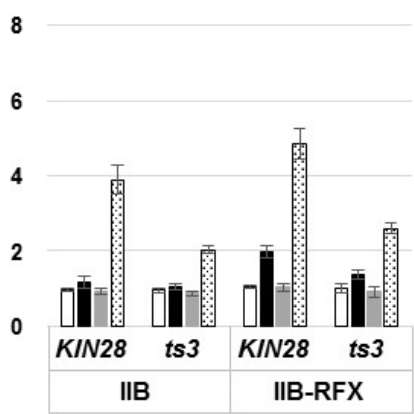

$\square$ IME2 $\square$ XMET17-GFP $\square$ MET2 囷 PMA1

Fig. 1. Effect of Kin28 inactivation on Mediator recruitment. KIN28 and kin28-ts3 cells expressing Cterminally TAP-tagged Med14 (a), Med17 (b) or Cdk8 (c) and containing xMET17-GFP at the chromosome and SUA7 (TFIIB) under the control of the glucose-repressed GAL1 promoter (PGAL1SUA7) were transformed with plasmids harboring IIB or IIB-RFX or MaxRFX. Cells were grown in glucose-containing synthetic minimal medium supplemented with $0.5 \mathrm{mM}$ methionine to early log phase at $26^{\circ} \mathrm{C}$, and shifted to $37^{\circ} \mathrm{C}$ for $45 \mathrm{~min}$. Association of Pol II (Rpb1 ChIP) and Mediator (TAP ChIP) with $x$ MET17-GFP, PMA1, MET2 and IME2 was measured after the shift at $37^{\circ} \mathrm{C}$ by ChIP with antibodies against Rpb1 or the TAP tag. MET2 is a methionine biosynthetic gene belonging to the same regulatory network as MET17 and IME2, a meiotic gene transcriptionally inactive in vegetative growth. DNA was quantitated by qPCR. Primers for IME2 are located in the middle of the ORF; primers for $x M E T 17-G F P$ are located at the 5' of the GFP ORF (upper graph) or at $x M E T 17$ promoter (lower graph); primer for MET2 and PMA1 are located at the 5' of the ORF (upper graph) or at the UAS (lower graph) (see Table S2). Occupancy is given relative to the value for IME2 in the wild type (see Materials and methods). Error bars indicate the average deviation of three independent experiments. 

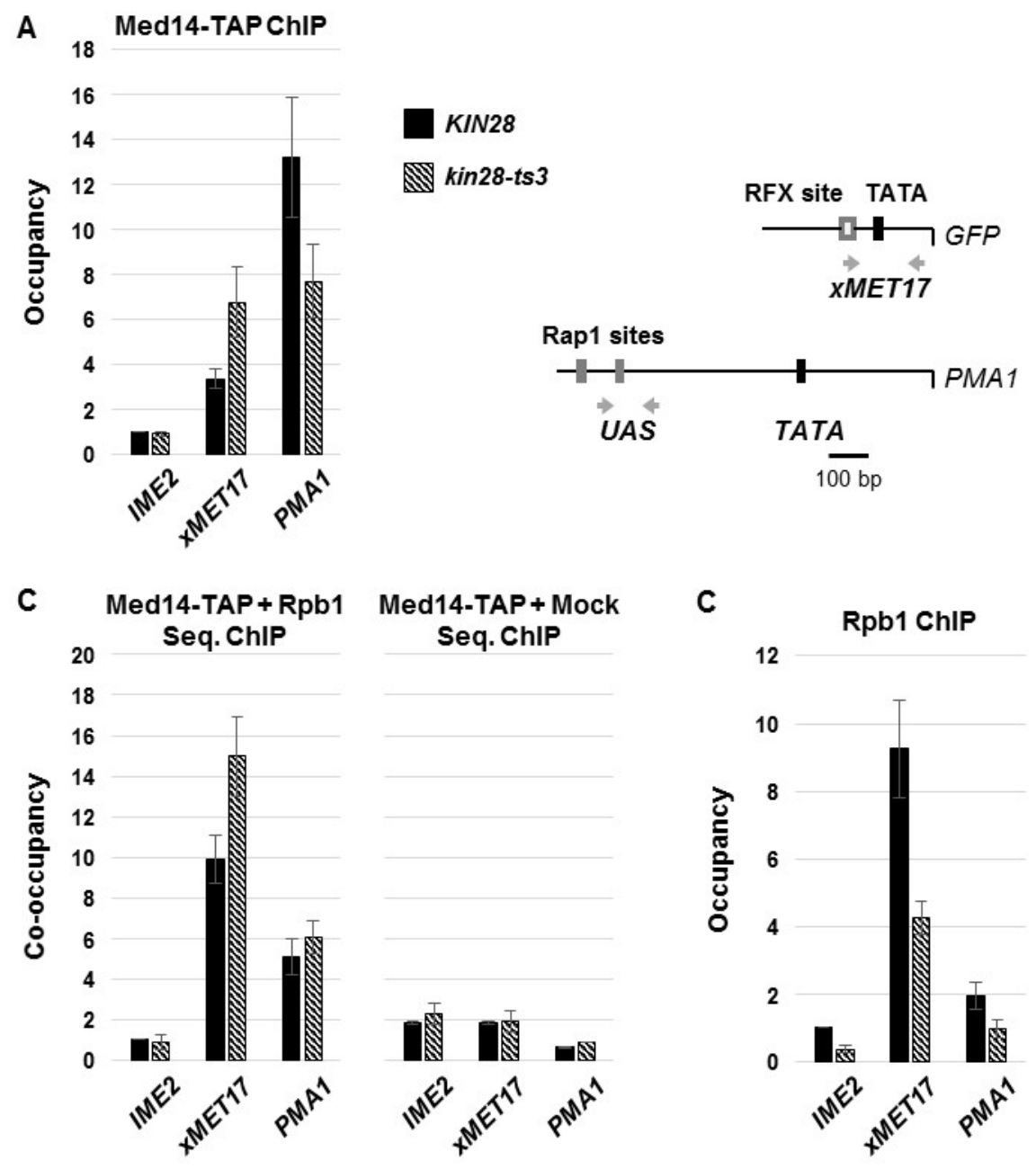

Fig. 2. Med14 and Pol II co-occupancy. Med14 TAP-tagged KIN28 and kin28-ts3 cells containing $x$ MET17-GFP integrated into the chromosome and PGAL1-SUA7 were transformed with pRS316-IIBRFX and grown as in Fig. 1 to early $\log$ phase at $26^{\circ} \mathrm{C}$ and shifted to $37^{\circ} \mathrm{C}$ for 30 min before fixation with formaldehyde. Chromatin extracts were subjected to a first ChIP using antibodies against Med14TAP (a), followed by a second ChIP using either antibodies against Pol II or no antibody (Mock) (b), or were subjected to single ChIP using antibodies against Rpb1 (c). DNA was quantitated by qPCR using specific primers for IME2, $x M E T 17$ promoter and PMA1 UAS region (arrows indicate the positions of the primers on the schematic). Co-occupancy is given relative to the value for IME2 in the wild type (see Materials and methods). Error bars indicate the average deviation of three independent experiments. 


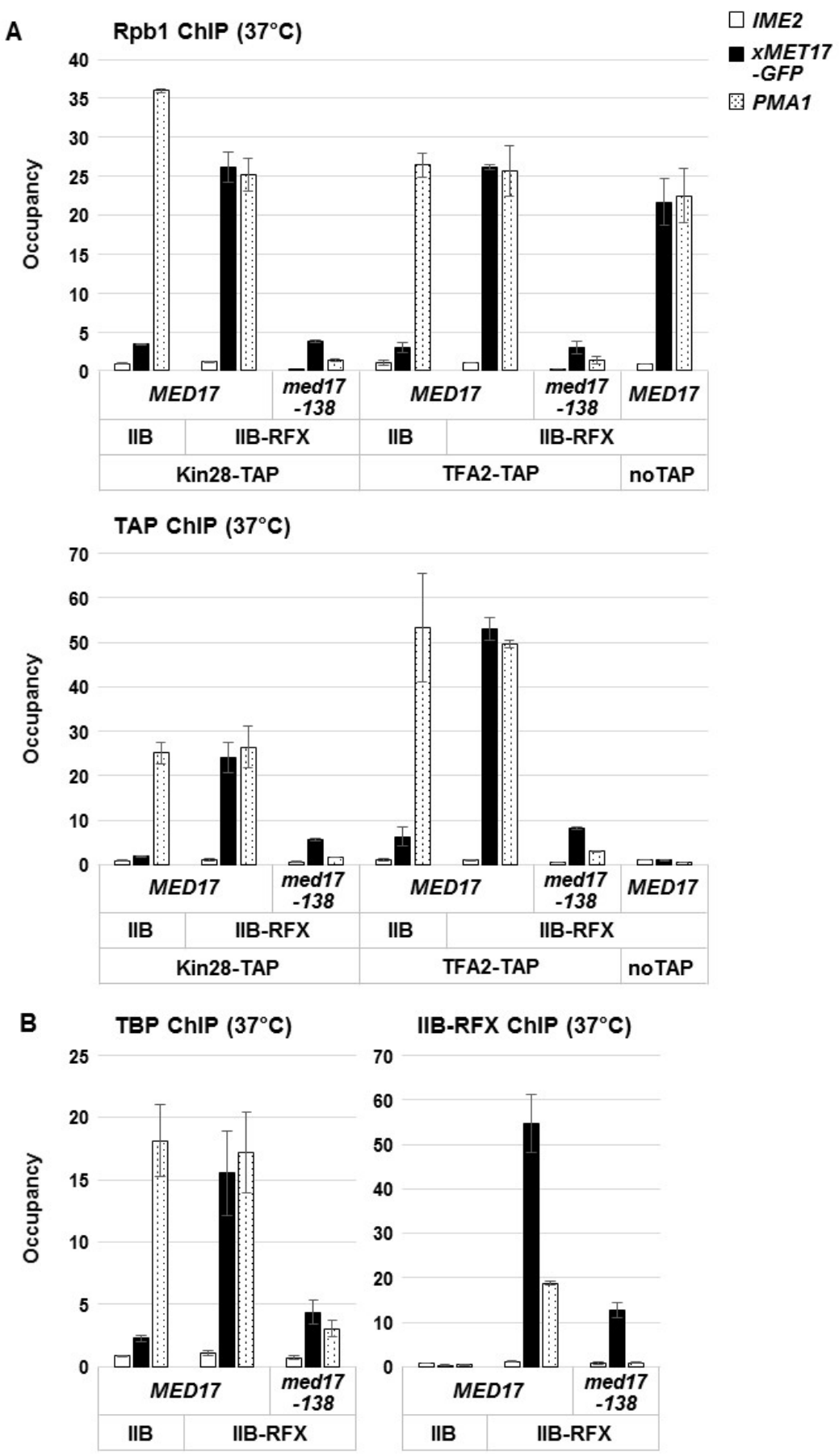

Fig. 3. Effect of med17-138 temperature-sensitive allele on assembly of the Pol II machinery. MED17 and med17-138 cells both expressing Kin28-TAP, TFA2-TAP or no tagged protein and containing PGAL1-SUA7 and XMET17-GFP at the chromosome were transformed with pRS316-IIB or pRS316IIB-RFX. Cells were grown as in Fig. 1 to early $\log$ phase at $28^{\circ} \mathrm{C}$ and shifted to $37^{\circ} \mathrm{C}$ for $45 \mathrm{~min}$ before fixation with formaldehyde. Associations of Pol II, Kin28-TAP or TFA2-TAP (a), and TBP or IIB-RFX (b) with the indicated genes were measured by ChIP with antibodies against Rpb1, the TAP-tag, TBP or RFX. The TBP ChIP was performed on the Kin28-TAP extracts and the RFX ChIP on the TFA2-TAP extracts. DNA was quantitated by qPCR using primers for IME2 as background control and primers for the 5'ORF (Rpb1 ChIP) or the promoter region (Kin28-TAP, TFA2-TAP, TBP and IIB-RFX ChIP) of $x M E T 17-G F P$ and PMA1. Occupancy is given relative to the value for IME2 in the wild type (see Materials and methods). Error bars indicate the average deviations of three independent experiments. 
A

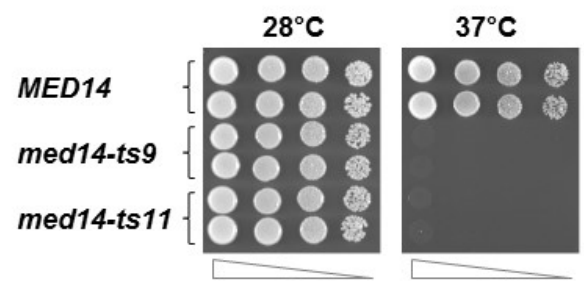

B

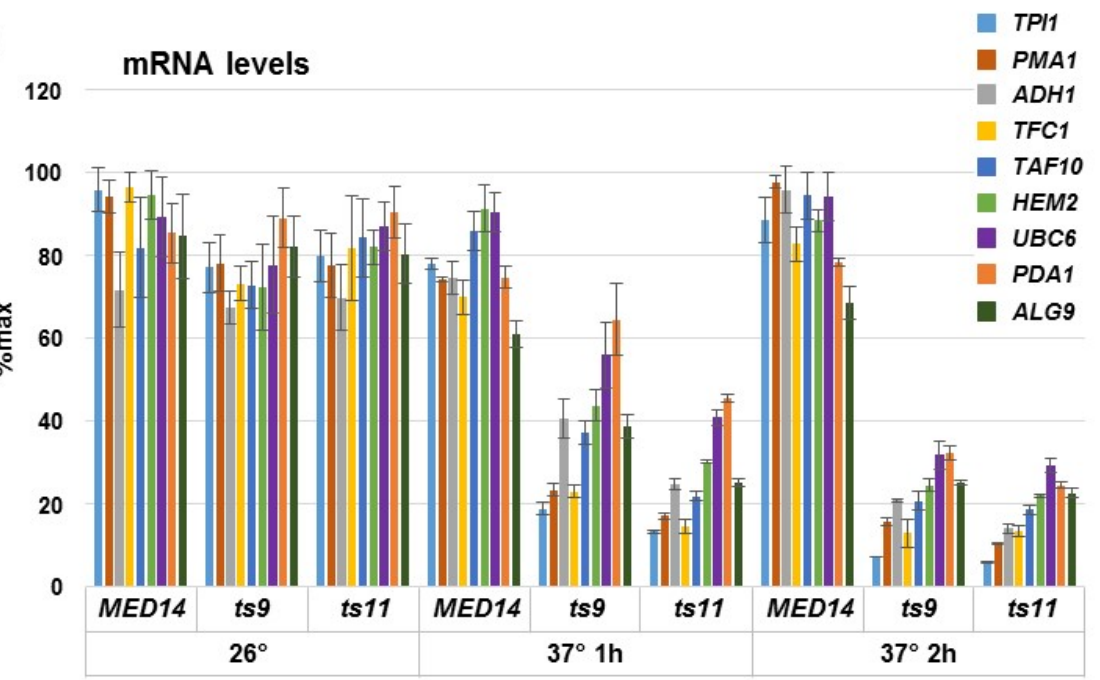

C

Rpb1 ChIP $\left(37^{\circ} \mathrm{C}\right)$

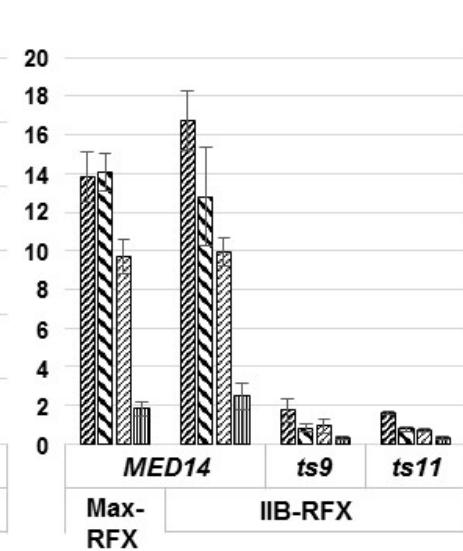

$\square$ IME2

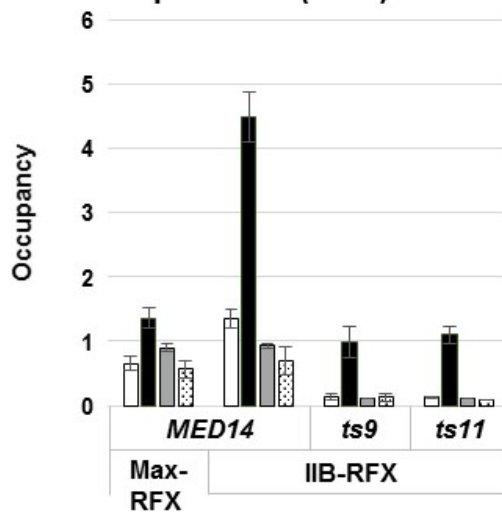

- XMET17

$\square$ MET2

중 GAL1

$A D H 1$

$\triangle$ PMA1

$T P 11$

血 PDA1

Fig. 4. Analysis of med14 temperature-sensitive mutants. (a) Phenotypic analyses. The med14-ts9, med14-ts11 mutants and isogenic wild-type strain were spotted in duplicate on rich medium and incubated at $28^{\circ} \mathrm{C}$ or $37^{\circ} \mathrm{C}$ for 2 days. The most concentrated spots correspond to $0.2 \times 10^{7}$ cells $/ \mathrm{ml}$. (b) Transcriptional analysis. Strains used in (a) were grown to early $\log$ phase at $26^{\circ} \mathrm{C}$ in rich medium and shifted to $37^{\circ} \mathrm{C}$. Total RNA was extracted before $\left(26^{\circ} \mathrm{C}\right)$ and after the temperature shift $\left(37^{\circ} \mathrm{C}, 1 \mathrm{~h}\right.$ and $2 \mathrm{~h}$ ). RT-qPCR analysis was performed on the indicated genes and $25 \mathrm{~S}$ rRNA for normalization. Values represent the average of two independent experiments expressed as a percentage of the maximum. Error bars indicate average deviations. The genes are involved in the following processes: TPI1, glycolysis; PMA1, plasma membrane proton transport; $A D H 1$, alcohol biosynthesis; TFC1, RNA pol III transcription; TAF10, RNA pol II transcription; HEM2, heme biosynthesis; UBC6, ubiquitin conjugation; PDA1, acetyl-CoA biosynthesis; ALG9, N-glycosylation. (c) Rpb1 recruitment. MED14, med14-ts9 and med14-ts 11 containing $x M E T 17-G F P$ at the chromosome and transformed with pRS315Max-RFX or pRS315-IIB-RFX were grown as in Fig. 1 to early $\log$ phase at $26^{\circ} \mathrm{C}$ and shifted to $37^{\circ} \mathrm{C}$ for 105 min before formaldehyde crosslinking. Rpb1 association with the genes indicated was measured by ChIP. DNA was analyzed by qPCR using primers specific for the coding regions. Occupancy is given relative to the value for IME2 in the wild type (see Materials and methods). Error bars indicate average deviations of four independent experiments. 

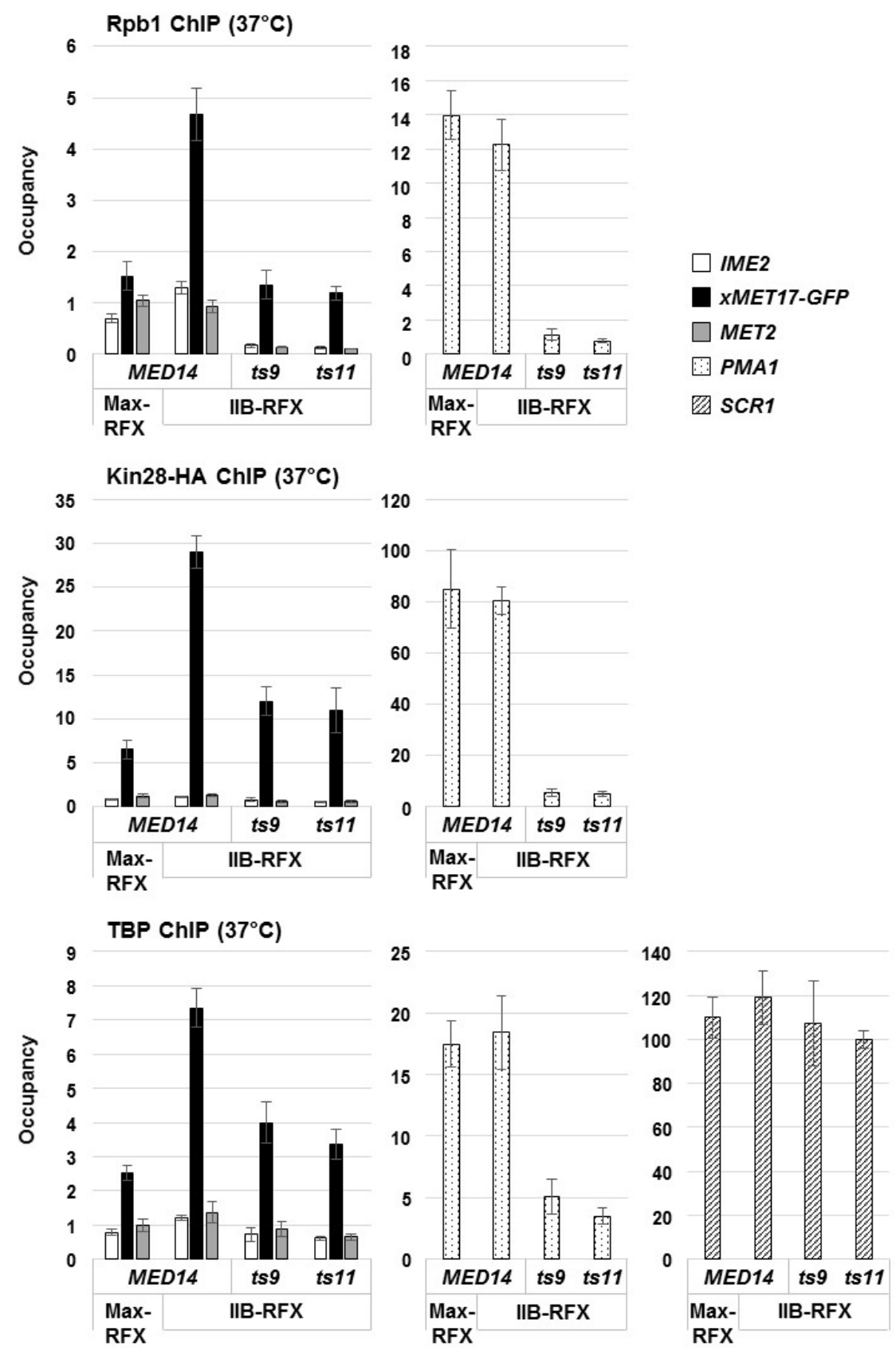

Fig. 5. Effect of med14-ts9 and med14-ts11 temperature-sensitive alleles on assembly of the Pol II machinery. MED14, med14-ts9 and med14-ts11 cells both expressing Kin28-TAP and containing

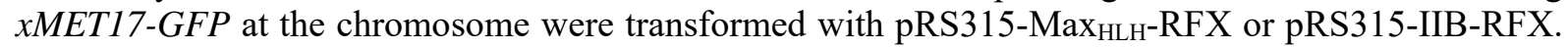
Cells were grown as in Fig. 1 to early $\log$ phase at $28^{\circ} \mathrm{C}$ and shifted to $37^{\circ} \mathrm{C}$ for 100 min before fixation with formaldehyde. Association of Pol II (upper graph), Kin28-HA (middle graph), and TBP (lower graph) with the indicated genes was measured by ChIP with antibodies against Rpb1, the HA tag, or TBP. DNA was quantitated by qPCR using primers for the ORF IME2 as background control and for the ORF (Rpb1 ChIP) or the promoter region (Kin28-TAP and TBP) of $x M E T 17-G F P, M E T 2$, and PMA1. Primers for SCR1 was also used in the case of TBP ChIP (SCR1 is transcribed by RNA Pol III and serves here as a positive control). Occupancy is given relative to the value for IME2 in the wild type (see Materials and methods). Error bars indicate the average deviations of three independent experiments. 


\section{Supplementary material for:}

Mediator, known as a coactivator, can act in transcription initiation in an activator-independent manner in vivo

Hélène Jean-Jacques, Siew Lay Poh and Laurent Kuras

Institute for Integrative Biology of the Cell (I2BC), CEA, CNRS, Univ. Paris-Sud, Université Paris-Saclay, Gif-sur-Yvette, France

Table S1. Saccharomyces cerevisiae strains

Table S2. Sequence of primers used in the ChIP experiments

Table S3. Sequence of primers used in the RT-qPCR experiments

Fig. S1. $x M E T 17-G F P$ transcriptional activation by TFIIB-RFX in KIN28 and kin28-ts3 cells.

Fig. S2. Pol II recruitment and transcription analysis in KIN28 and kin28-ts3 strains expressing TAP-tagged Med14 or Med 17 grown at $26^{\circ} \mathrm{C}$.

Fig. S3. Controls for ChIP enrichment of $x M E T 17-G F P$ in KIN28 and kin28-ts3.

Fig. S4. Transcription analysis in MED17 and med17-138 strains expressing TAP-tagged Kin28, TFA2 or Med14 grown at $26^{\circ} \mathrm{C}$.

Fig. S5. Transcription activation by TFIIB-RFX in MED14, med14-ts9 and med14-ts11 strains.

Fig. S6. Effect of med17-138 on Med14 recruitment. 
Table S1. Saccharomyces cerevisiae strains

\begin{tabular}{|c|c|}
\hline Strain & Relevant genotype \\
\hline Y667 & $\begin{array}{l}\text { ade2-1, lys2-801, ura3-52, trp1- } \Delta 63 \text {, his3- } \Delta 200, \text { leu2- } \Delta 1, \text { med14::KanMX4/ } \\
\text { pVV208-MED14 (URA3, CEN) }\end{array}$ \\
\hline Y1161 & KIN28, met17::P $P_{T E F}-b l e-T_{T E F}$-xMET17-GFP, sua7::KanMX6-PGAL1-SUA7 \\
\hline Y1162 & kin28-ts3, met17:: $P_{T E F}-b l e-T_{T E F}$-xMET17-GFP, sua7::KanMX6-PGAL1-SUA7 \\
\hline Y1166 & KIN28, MED17-TAP-KITRP1, met17::P $P_{T E F}-b l e-T_{T E F}-\mathrm{xMET17-GFP,} \mathrm{sua7::KanMX6-PGAL1-SUA7}$ \\
\hline Y1167 & 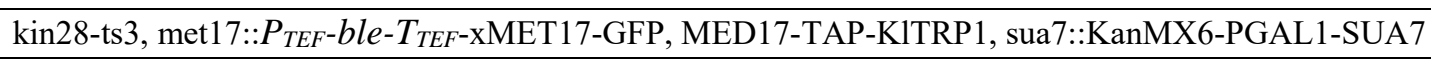 \\
\hline Y1170 & KIN28, MED14-TAP-K1TRP1, met17:: $P_{T E F}-b l e-T_{T E F}-\mathrm{xMET17-GFP,} \mathrm{sua7::KanMX6-PGAL1-SUA7}$ \\
\hline Y1172 & kin28-ts3, MED14-TAP-KITRP1, met17:: $P_{T E F}-$ ble- $T_{T E F}$-XMET17-GFP, sua7::KanMX6-PGAL1-SUA7 \\
\hline Y1178 & $\begin{array}{l}\text { KIN28-TAP-K1TRP1, met17::P } P_{T E F}-b l e-T_{T E F} \text {-XMET17-GFP, sua7::KanMX6-PGAL1-SUA7, } \\
\text { med17D::His3MX6/RY2844 (MED17, LEU2, CEN) }\end{array}$ \\
\hline Y1182 & $\begin{array}{l}\text { KIN28-TAP-KITRP1, met17::P } P_{T E F}-\text { ble- } T_{T E F} \text {-XMET17-GFP, sua7::KanMX6-PGAL1-SUA7, } \\
\text { med17 }:: \text { His3MX6/RY2882 (med17-138, LEU2, CEN) }\end{array}$ \\
\hline Y1195 & $\begin{array}{l}\text { met17::P } P_{\text {TEF }}-b l e-T_{\text {TEF-XMET17-GFP, sua 7::KanMX6-PGAL1-SUA7, med17 }}:: \text { His3MX6/ } \\
\text { RY2844 (MED17, LEU2, CEN) }\end{array}$ \\
\hline Y1212 & $\begin{array}{l}\text { TFA2-TAP-KITRP1, met17::P } P_{\text {TEF }} \text {-ble- } T_{\text {TEF-XMET17-GFP, sua7::KanMX6-PGAL1-SUA7, }} \\
\text { med17 } \Delta: \text { His3MX6/RY2844 (MED17, LEU2, CEN) }\end{array}$ \\
\hline Y1217 & $\begin{array}{l}\text { TFA2-TAP-KITRP1, met17::P } P_{T E F}-b l e-T_{T E F}-\mathrm{xMET17-GFP,} \mathrm{sua7::KanMX6-PGAL1-SUA7,} \\
\text { med17L::His3MX6/RY2882 (med17-138, LEU2, CEN) }\end{array}$ \\
\hline Y1220 & $\begin{array}{l}\text { MED14-TAP-KITRP1, met17::P } P_{\text {TEF }} \text {-ble-T } T_{\text {TEF-XMET17-GFP, sua7::KanMX6-PGAL1-SUA7, }} \\
\text { med17 }:: \text { His3MX6/RY2844 (MED17, LEU2, CEN) }\end{array}$ \\
\hline Y1223 & $\begin{array}{l}\text { MED14-TAP-KITRP1, met17::P } P_{\text {TEF }}-b l e-T_{\text {TEF }} \text {-XMET17-GFP, sua7::KanMX6-PGAL1-SUA7, } \\
\text { med17 }:: \text { His3MX6/RY2882 (med17-138, LEU2, CEN) }\end{array}$ \\
\hline Y1346 & $\begin{array}{l}\text { ade2-1, lys2-801, ura3-52, trp1- } \Delta 63, \text { his3- } \Delta 200, \text { leu2- } \Delta 1, \text { met17:: } P_{T E F}-b l e-T_{T E F}-\mathrm{XMET} 17-\mathrm{GFP}, \\
\text { med14 } \Delta:: \text { KanMX4/pVV208-MED14 (URA3, CEN) }\end{array}$ \\
\hline Y1356 & met17::P $P_{T E F}-b l e-T_{T E F}-\mathrm{xMET17-GFP,} \mathrm{med14D::KanMX4/pVV204-MED14} \mathrm{(TRP1,} \mathrm{CEN)}$ \\
\hline Y1360 & 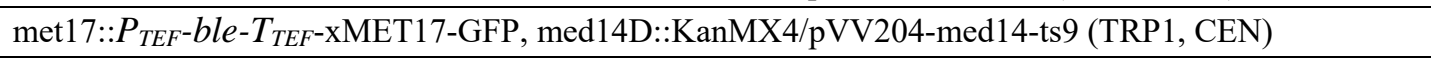 \\
\hline Y1362 & met17::P $P_{T E F}-b l e-T_{T E F}-\mathrm{xMET17-GFP,} \mathrm{med14D::KanMX4/pVV204-med14-ts11} \mathrm{(TRP1,} \mathrm{CEN)}$ \\
\hline Y1365 & $\begin{array}{l}\text { KIN28-3HA-HIS3MX6, met17:: } P_{\text {TEF }}-\text { ble- } T_{\text {TEF- }} \text {-XMET17-GFP, } \\
\text { med14D::KanMX4/pVV208-MED14 (URA3, CEN) }\end{array}$ \\
\hline Y1370 & $\begin{array}{l}\text { KIN28-3HA-HIS3MX6 met17::P } P_{\text {TEF }}-b l e-T_{\text {TEF }} \text {-XMET17-GFP, } \\
\text { med14D::KanMX4/pVV204-MED14 (TRP1, CEN) }\end{array}$ \\
\hline Y1372 & 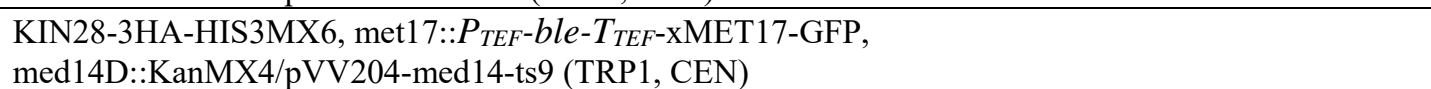 \\
\hline Y1374 & $\begin{array}{l}\text { KIN28-3HA-HIS3MX6, met17::P } P_{\text {TEF }} \text {-ble- } T_{\text {TEF- }} \text {-XMET17-GFP, } \\
\text { med14D::KanMX4/pVV204-med14-ts11 (TRP1, CEN) }\end{array}$ \\
\hline Y1386 & KIN28, CDK8-TAP-K1TRP1, met17::P $P_{T E F-b l e-T_{T E F} \text {-XMET17-GFP, sua7::KanMX6-PGAL1-SUA7 }}$ \\
\hline Y1388 & kin28-ts3, CDK8-TAP-KITRP1, met17::P $P_{T E F}-b l e-T_{T E F}-\mathrm{xMET17-GFP,} \mathrm{sua7::KanMX6-PGAL1-SUA7}$ \\
\hline
\end{tabular}


Table S2. Sequence of primers used in the ChIP experiments

\begin{tabular}{|c|c|c|c|c|}
\hline Gene (orf size) & Oligo\# & Strand & $\begin{array}{l}\text { Position } \\
\text { (to ATG) }\end{array}$ & Sequence 5'->3' \\
\hline \multirow{2}{*}{$\begin{array}{l}\text { ACT1 } \\
\text { (orf=1437bp) }\end{array}$} & 1575 & Fwd & +306 & ATTATATGTTTAGAGGTTGCTGCTTTGG \\
\hline & 1576 & Rev & +590 & CAATTCGTTGTAGAAGGTATGATGCC \\
\hline \multirow{2}{*}{$\begin{array}{l}\text { ADH1 } \\
\text { (orf=1047bp) }\end{array}$} & 1178 & Fwd & +27 & GTTATCTTCTACGAATCCCACGG \\
\hline & 1179 & Rev & +190 & CTAATGGTAGCTTAACTGGC \\
\hline \multirow{2}{*}{$\begin{array}{l}\text { GAL1 } \\
\text { (orf=1587bp) }\end{array}$} & 344 & Fwd & +12 & TCATTCAGAAGAAGTGATTGTACCTG \\
\hline & 345 & Rev & +150 & CGATCTAGCAACAAAATCCGGTTTAG \\
\hline \multirow{2}{*}{$\begin{array}{l}\text { IME2 } \\
\text { (orf=1938bp) }\end{array}$} & 2014 & Fwd & +1023 & TGTAGAAGGTTCATCGTTGGATCA \\
\hline & 2015 & Rev & +1124 & GCCCTTTCATTAGGATCCCATCTT \\
\hline \multirow[t]{2}{*}{ MET2 UAS } & 111 & Fwd & -395 & ATTTCTTGCTATTGTTAGTGGCTCC \\
\hline & 405 & Rev & -242 & GGTGTGTGCCAAATCCAAACGATTA \\
\hline \multirow{2}{*}{$\begin{array}{l}\text { MET2 } \\
\text { (orf=1461bp) }\end{array}$} & 413 & Fwd & +205 & GTAATTTGTCATGCCTTGACTGGGTC \\
\hline & 414 & Rev & +379 & ATCTAACGCCCGTCTCCTCATTTAT \\
\hline \multirow{2}{*}{$\begin{array}{l}\text { PDA1 } \\
\text { (orf=1263bp) }\end{array}$} & 1583 & Fwd & +704 & ATTTGCCCGTCGTGTTTTGCTGTG \\
\hline & 1584 & Rev & +988 & TATGCTGAATCTCGTCTCTAGTTCTGTAGG \\
\hline \multirow[t]{2}{*}{ PMA1 UAS } & 1784 & Fwd & -846 & AACAAACCCGGTCTCGAAG \\
\hline & 1785 & Rev & -691 & GAAGTGCCGCATTAGGAAAT \\
\hline \multirow[t]{2}{*}{ PMA1 TATA } & 2030 & Fwd & -378 & GATGGTGGGTACCGCTTATG \\
\hline & 2031 & Rev & -235 & TTGGTGTTATAGGAAAGAAAGAGAAAA \\
\hline \multirow{2}{*}{$\begin{array}{l}\text { PMA1 5'-orf } \\
\text { (orf=2757bp) }\end{array}$} & 1963 & Fwd & +49 & TCAGCTCATCAGCCAACTCAAG \\
\hline & 1964 & Rev & +172 & CGTCGACACCGTGATTAGATTG \\
\hline \multirow{2}{*}{$\begin{array}{l}\text { PMA1 3'-orf } \\
\text { (orf=2757bp) }\end{array}$} & 1965 & Fwd & +2581 & TACTGTCGTCCGTGTCTGGATCT \\
\hline & 1966 & Rev & +2677 & CCTTCATTGGCTTACCGTTCA \\
\hline \multirow[t]{2}{*}{ SCR1 } & 1198 & Fwd & +2 & GGCTGTAATGGCTTTCTGG \\
\hline & 1199 & Rev & +124 & GTGCGGAATAGAGAACTATCC \\
\hline \multirow{2}{*}{$\begin{array}{l}\text { TPI1 } \\
\text { (orf=747bp) }\end{array}$} & 370 & Fwd & +472 & ACTAACGTCGTTGTCGCTTACGAACC \\
\hline & 371 & Rev & +688 & AGAAACCATCGACATCAGCCTTGT \\
\hline \multirow[t]{2}{*}{ xMET17 promoter } & 1512 & Fwd & -227 & TTGCCTAGCAACTACATATGGTCACC \\
\hline & 1513 & Rev & -23 & TTGTATCTATGTATCTGACGACCCTG \\
\hline \multirow[t]{2}{*}{ xMET17-GFP TSS } & 2032 & Fwd & -60 & CTTCGTGTAATACAGGGTCGTCAGAT \\
\hline & 2033 & Rev & +38 & GGGACAACACCAGTGAATAATTCTTC \\
\hline \multirow{2}{*}{$\begin{array}{l}\text { xMET17-GFP 5'-orf } \\
\text { (orf=717bp) }\end{array}$} & 2016 & Fwd & +36 & CCCAATTTTGGTTGAATTAGATGG \\
\hline & 2017 & Rev & +170 & CATGGAACTGGCAATTTACCAGTA \\
\hline \multirow{2}{*}{$\begin{array}{l}\text { xMET17-GFP 3'-orf } \\
\text { (orf=717bp) }\end{array}$} & 1424 & Fwd & +404 & GAAGATGGTAACATTTTAGGTCACAAA \\
\hline & 1425 & Rev & +586 & GTAACAAGACTGGACCATCACCAAT \\
\hline
\end{tabular}


Table S3. Sequence of primers used in the RT-qPCR experiments

\begin{tabular}{|c|c|c|c|c|}
\hline Gene & Oligo \# & Strand & $\begin{array}{l}\text { Position } \\
\text { (to ATG) }\end{array}$ & Sequence 5'->3' \\
\hline \multirow[t]{2}{*}{$25 \mathrm{~S}$ rDNA } & 394 & Fwd & +624 & GGTTATATGCCGCCCGTCTTGA \\
\hline & 395 & Rev & +800 & CCCAACAGCTATGCTCTTACTC \\
\hline \multirow[t]{2}{*}{ ACT1 } & 1575 & Fwd & +306 & ATTATATGTTTAGAGGTTGCTGCTTTGG \\
\hline & 1576 & Rev & +590 & CAATTCGTTGTAGAAGGTATGATGCC \\
\hline \multirow[t]{2}{*}{ ADH1 } & 1178 & Fwd & +27 & GTTATCTTCTACGAATCCCACGG \\
\hline & 1179 & Rev & +190 & CTAATGGTAGCTTAACTGGC \\
\hline \multirow[t]{2}{*}{ ALG9 } & 1577 & Fwd & +1091 & CACGGATAGTGGCTTTGGTGAACAATTAC \\
\hline & 1578 & Rev & +1252 & TATGATTATCTGGCAGCAGGAAAGAACTTGGG \\
\hline \multirow[t]{2}{*}{ GFP } & 382 & Fwd & +26 & CTGGTGTTGTCCCAATTTTGGTTG \\
\hline & 383 & Rev & +215 & GCAAAACATTGAACACCATAACCGA \\
\hline \multirow[t]{2}{*}{ HEM2 } & 1579 & Fwd & +128 & TTCCGCTATTCATCTCCGATAATCCAG \\
\hline & 1580 & Rev & +399 & ACAGACATCGCAAATAATATACAGTTCAGG \\
\hline \multirow[t]{2}{*}{ PDA1 } & 1583 & Fwd & +704 & ATTTGCCCGTCGTGTTTTGCTGTG \\
\hline & 1584 & Rev & +988 & TATGCTGAATCTCGTCTCTAGTTCTGTAGG \\
\hline \multirow[t]{2}{*}{ PMA1 } & 1963 & Fwd & +49 & TCAGCTCATCAGCCAACTCAAG \\
\hline & 1964 & Rev & +172 & CGTCGACACCGTGATTAGATTG \\
\hline \multirow[t]{2}{*}{ TAF10 } & 1589 & Fwd & +389 & ATATTCCAGGATCAGGTCTTCCGTAGC \\
\hline & 1590 & Rev & +530 & GTAGTCTTCTCATTCTGTTGATGTTGTTGTTG \\
\hline \multirow[t]{2}{*}{ TFC1 } & 1591 & Fwd & +1056 & GCTGGCACTCATATCTTATCGTTTCACAATGG \\
\hline & 1592 & Rev & +1278 & GAACCTGCTGTCAATACCGCCTGGAG \\
\hline \multirow[t]{2}{*}{ TPI1 } & 370 & Fwd & +472 & ACTAACGTCGTTGTCGCTTACGAACC \\
\hline & 371 & Rev & +688 & AGAAACCATCGACATCAGCCTTGT \\
\hline \multirow[t]{2}{*}{ UBC6 } & 1593 & Fwd & +286 & GATACTTGGAATCCTGGCTGGTCTGTCTC \\
\hline & 1594 & Rev & +557 & AAAGGGTCTTCTGTTTCATCACCTGTATTTGC \\
\hline \multirow{2}{*}{ xMET17-GFP } & 382 & Fwd & +26 & CTGGTGTTGTCCCAATTTTGGTTG \\
\hline & 383 & Rev & +215 & GCAAAACATTGAACACCATAACCGA \\
\hline
\end{tabular}



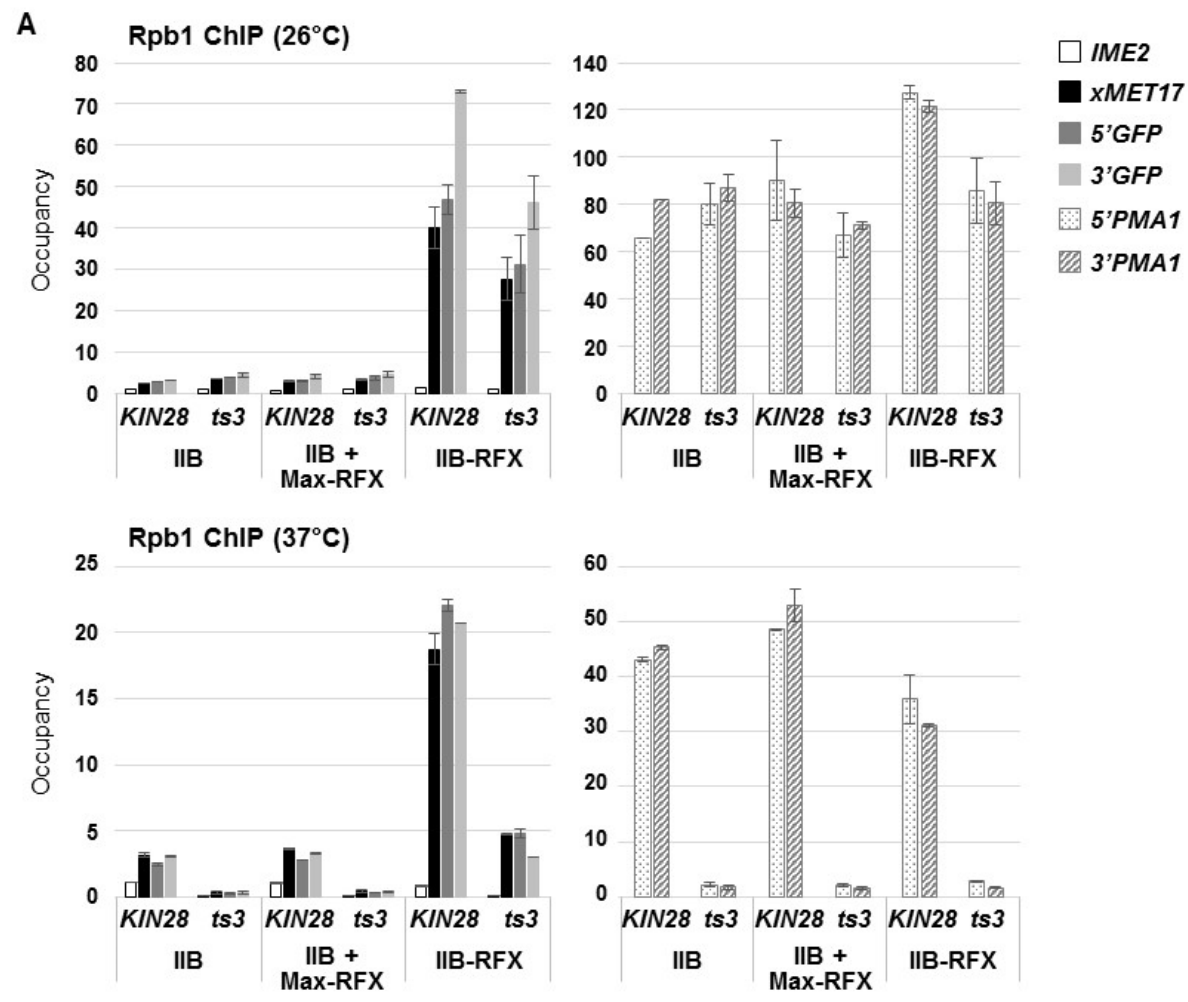

B

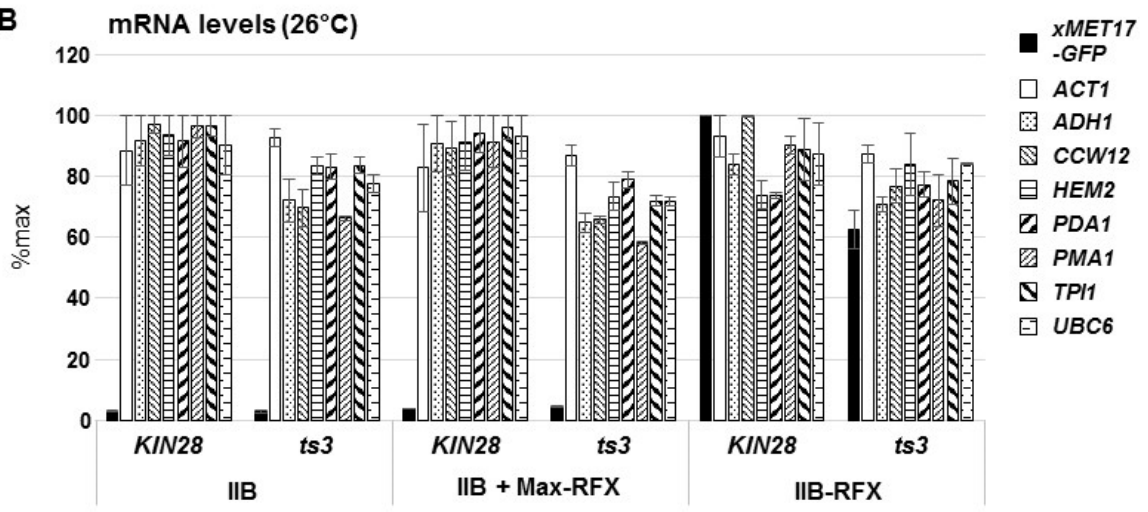

Fig. S1. $x M E T 17-G F P$ transcriptional activation by TFIIB-RFX in KIN28 and kin28-ts3 cells. (A) kin28ts3 and KIN28 strains containing $x M E T 17-G F P$ at the chromosome and expressing TFIIB under the control of the GAL1 promoter were transformed with pRS313-IIB + pRS315, pRS313-IIB + pRS315-MaxRFX or pRS313 + pRS315-IIB-RFX. Cells grown to early log phase at $26^{\circ} \mathrm{C}$ in glucose-containing minimal synthetic medium supplemented with $0.5 \mathrm{mM}$ methionine, and shifted to $37^{\circ} \mathrm{C}$. Pol II occupancy was measured by ChIP before and 60 min after the shift. DNA was analyzed by qPCR using primers specific for IME2, $x$ MET17 promoter, and GFP or PMA1 5'- and 3'-ORF. Occupancy is given relative to the value for IME2 in the wild type (see Materials and methods). Error bars indicate standard deviations from two independent experiments. (B) RNA levels for $x M E T 17-G F P$ and various housekeeping genes in cells taken before the shift to $37^{\circ} \mathrm{C}$ from the cultures used in (A). Values were measured by RT-qPCR and normalized to $25 \mathrm{~S}$ rRNA levels. Error bars indicate the average deviation of two independent experiments. 


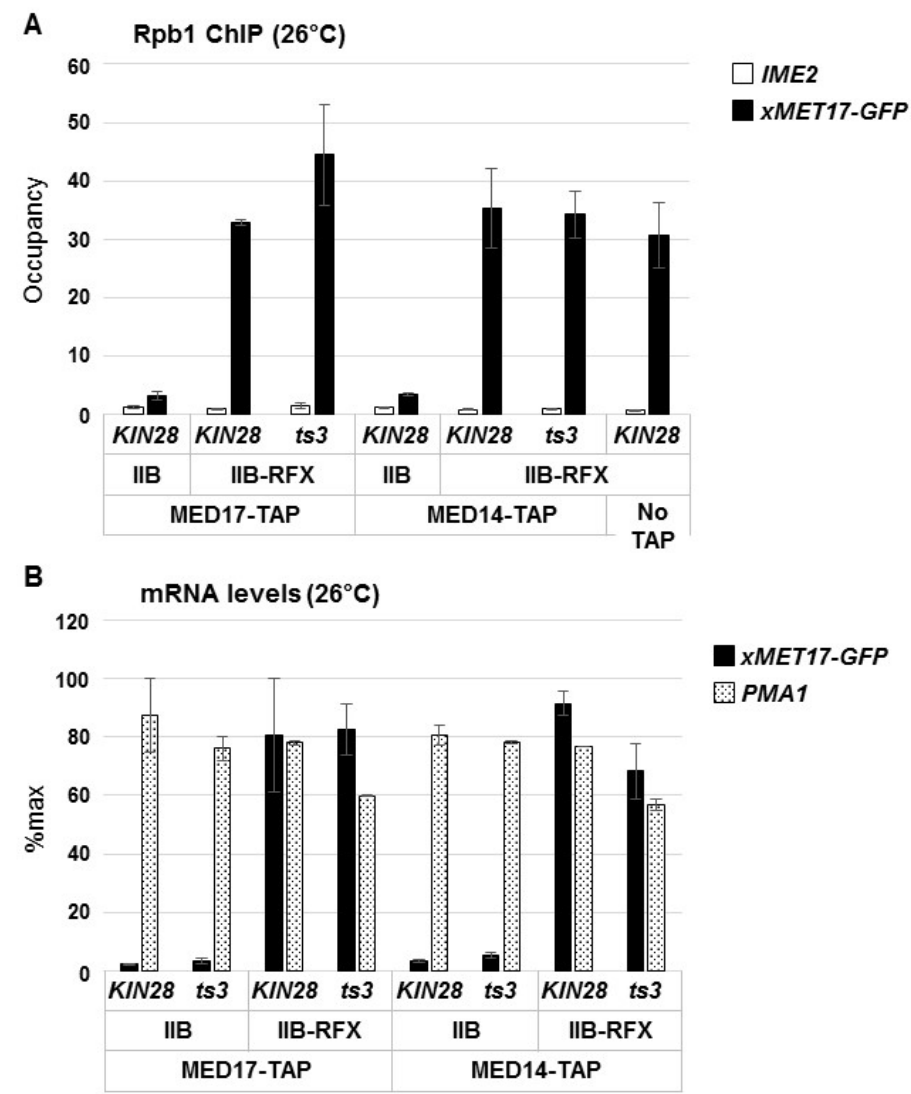

Fig. S2. Pol II recruitment and transcription analysis in KIN28 and kin28-ts3 strains expressing TAP-tagged Med14 or Med17 grown at $26^{\circ} \mathrm{C}$. Cells from the cultures in Fig. 1 were taken before the shift to $37^{\circ} \mathrm{C}$ and either fixed with formaldehyde at $26^{\circ} \mathrm{C}$ or frozen in liquid nitrogen. (A) Chromatin extracts prepared from the cells fixed with formaldehyde were subjected to ChIP with antibodies against Rpb1. DNA was analyzed by qPCR using primers specific for $x M E T 17-G F P$ or IME2 ORF. Occupancy levels are given relative to the average value obtained for IME2 in the wild type extracts. Error bars indicate the average deviation from two independent experiments. (B) Total RNA was extracted from the cells frozen in liquid nitrogen and RNA levels for $x M E T 17-G F P$ and PMA1 were measured by RT-qPCR. Values were normalized to $25 \mathrm{~S}$ rRNA levels and represent the average of two independent experiments expressed as a percentage of the maximum. Error bars indicate the average deviation. 


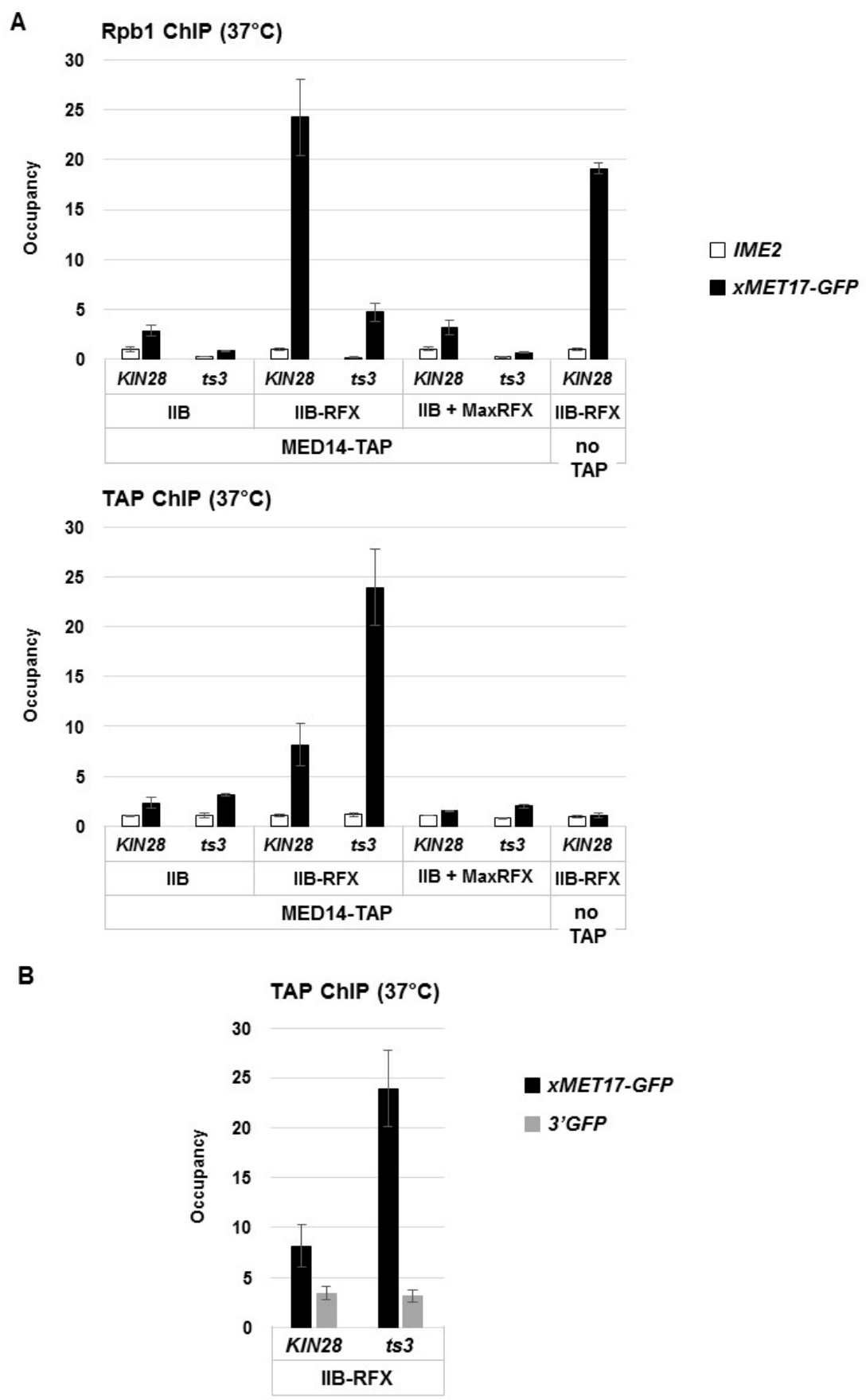

Fig. S3. Controls for ChIP enrichment of $x M E T 17-G F P$ in KIN28 and kin28-ts3. (A) The ChIP results obtained with the Med14-TAP strains in Fig. 1 were supplemented with an isogenic untagged strain expressing IIB-RFX that was processed in parallel. (B) Immunoprecipitates from the KIN28 and kin28-ts3 Med14-TAP strains expressing IIB-RFX used in Fig. 1 were analyzed using primers specific for the 3'end of GFP ORF. 


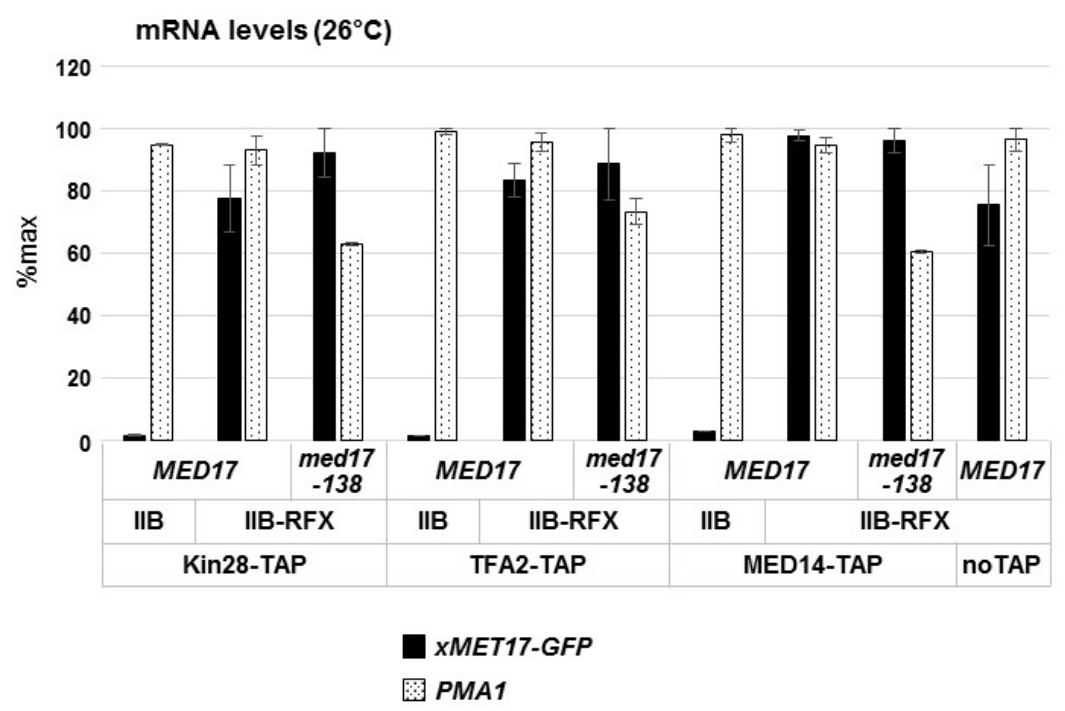

Fig. S4. Transcription analysis in MED17 and med17-138 strains expressing TAP-tagged Kin28, TFA2 or Med14 grown at $26^{\circ} \mathrm{C}$. RNA levels for $x M E T 17-G F P$ and PMA1 in cells taken before the shift to $37^{\circ} \mathrm{C}$ from the cultures used in Fig. 3 and 4. Values were measured by RT-qPCR and normalized to 25S rRNA levels. Values represent the average of two independent experiments expressed as a percentage of the maximum, and error bars indicate the average deviation. 


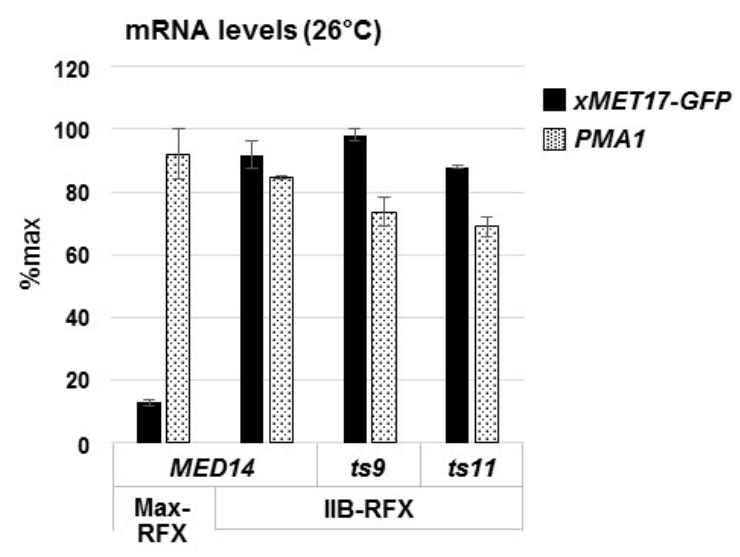

Fig. S5. Transcription activation by TFIIB-RFX in MED14, med14-ts9 and med14-ts11 strains. Total RNA was extracted from cell aliquots taken in experiment in Fig. $4 \mathrm{C}$ during growth at $26^{\circ} \mathrm{C}$. RT-qPCR analysis was performed on GFP and PMA1 as well as on 25S rRNA for normalization. Values represent the average of two independent experiments expressed as a percentage of the maximum, and error bars indicate the average deviation. 


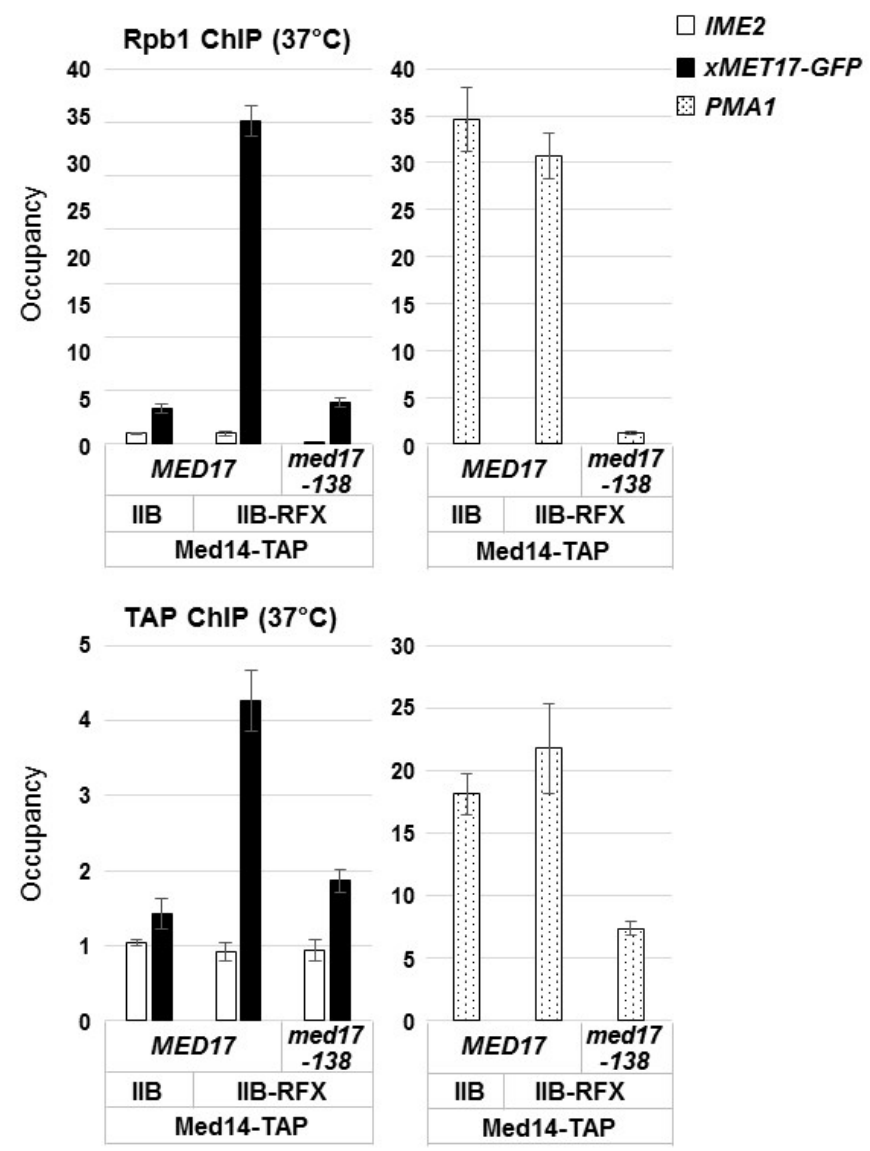

Fig. S6. Effect of med17-138 on Med14 recruitment. MED17 and med17-138 cells both expressing Med14-TAP and containing PGAL1-SUA7 and XMET17-GFP at the chromosome were transformed with pRS316-IIB or pRS316-IIB-RFX. Cells were grown and fixed with formaldehyde as in Fig. 3. Association of Pol II (upper graph) and Med14-TAP (lower graph) with the indicated genes was measured by ChIP with antibodies against Rpb1 or the TAP tag. DNA was quantitated by qPCR using primers for IME2 and for the 5'ORF of $x$ MET17-GFP and PMA1 (Rpb1 ChIP), or the promoter region of $x$ MET17-GFP and the UAS region of PMA1 (Med14-TAP ChIP). Occupancy is given relative to the value for IME2 in the wild type (see Materials and methods). Error bars indicate the average deviations of three independent experiments. 